

\title{
L'exposition des travailleurs aux risques psychosociaux a-t-elle augmenté pendant la crise économique de 2008 ?
}

\author{
Roméo Fontaine, Pascale Lengagne, Damien Sauze
}

\section{To cite this version:}

Roméo Fontaine, Pascale Lengagne, Damien Sauze. L'exposition des travailleurs aux risques psychosociaux a-t-elle augmenté pendant la crise économique de 2008 ?. Economie et Statistique / Economics and Statistics, 2016, 486, pp.103 - 128. 10.3406/estat.2016.10692 . hal-01391617

\section{HAL Id: hal-01391617 \\ https://hal.science/hal-01391617}

Submitted on 3 Nov 2016

HAL is a multi-disciplinary open access archive for the deposit and dissemination of scientific research documents, whether they are published or not. The documents may come from teaching and research institutions in France or abroad, or from public or private research centers.
L'archive ouverte pluridisciplinaire HAL, est destinée au dépôt et à la diffusion de documents scientifiques de niveau recherche, publiés ou non, émanant des établissements d'enseignement et de recherche français ou étrangers, des laboratoires publics ou privés. 


\title{
L'exposition des travailleurs aux risques psychosociaux a-t-elle augmenté pendant la crise économique de 2008 ?
}

\author{
Roméo Fontaine *, Pascale Lengagne ** et Damien Sauze ***
}

À partir des deux vagues de l'enquête Santé et itinéraire professionnel (SIP), cet article étudie l'exposition des travailleurs aux risques psychosociaux (RPS) et son évolution entre 2006 et 2010, dans un contexte de crise économique.

Nous estimons par des modèles Probit la probabilité individuelle d'exposition aux différents RPS conditionnellement aux caractéristiques socioéconomiques des individus et aux caractéristiques de leur emploi. Si l'on observe une surexposition générale aux RPS chez les individus de 35 à 44 ans, ceux ayant un diplôme de niveau baccalauréat ou ceux exposés à des pénibilités physiques, les profils d'exposition apparaissent nettement différenciés selon le genre, la catégorie professionnelle et le secteur d'activité. L'identification de ces surexpositions et de ces profils doit permettre de mieux cibler les politiques de prévention sur ces populations qui ne sont pas toujours celles que l'on considère comme les plus fragiles sur le marché du travail.

On observe une augmentation générale de l'exposition aux RPS sur la période 2006-2010. L'augmentation la plus frappante concerne le manque de reconnaissance. Nous analysons ces évolutions selon les caractéristiques économiques des secteurs d'activité, l'existence d'un plan de licenciement au niveau de l'entreprise et les mobilités professionnelles des individus. Aucun lien significatif n'est à noter entre l'évolution de l'exposition aux RPS et les différences sectorielles d'exposition à la crise. En revanche, nous mettons en évidence une association significative entre l'existence d'un plan de licenciement au niveau de l'entreprise et l'aggravation de l'exposition aux RPS. Enfin, connaitre une mobilité professionnelle est associé à une réduction significative de l'exposition aux RPS. Du fait du caractère pro-cyclique des mobilités professionnelles externes, ce lien pourrait constituer un canal de transmission entre crise économique et exposition aux RPS.

Les jugements et opinions exprimés par les auteurs

n'engagent qu'eux mêmes, et non les institutions auxquelles ils appartiennent, ni a fortiori l'Insee.
Codes JEL : I10, J81, J28.

Mots clés : risques psychosociaux, crise économique, mobilité professionnelle, Enquête Santé et itinéraire professionnel.

** IRDES (Leng 6307 CNRS-U1200 Inserm-Université de Bourgogne) et IRDES (Romeo.Fontaine@u-bourgogne.fr)

$\star \star *$ LEDI $(1+2$

00 Inserm-Université de Bourgogne) (Damien.Sauze@u-bourgogne.fr)

Remerciements : la recherche présentée dans cet article a bénéficié du soutien financier de la Dares. Les auteurs souhaitent remercier tout particulièrement Hélène Sultan-Taïeb (UQAM), Chantal Cases (Ined), Nicolas De Riccardis (Drees) et Thomas Coutrot (Dares), pour leur relecture attentive et leurs suggestions sur les premières versions de cet article. Ils remercient également les deux rapporteurs anonymes pour leurs commentaires et apports importants pour la finalisation de la dernière version de ce travail. Les auteurs restent seuls responsables des limites et des erreurs qui peuvent subsister. 
$\mathbf{L}$ e collège d'expertise sur le suivi statistique des risques psychosociaux au travail (2011) définit les RPS comme des « risques pour la santé mentale, physique et sociale, engendrés par les conditions d'emploi et les facteurs organisationnels et relationnels susceptibles d'interagir avec le fonctionnement mental $\gg$. Les RPS occupent une place grandissante dans l'étude de l'impact des conditions de travail sur la santé des travailleurs, au-delà des risques physiques ou chimiques ou du décompte des accidents du travail et maladies professionnelles. Ils recouvrent plusieurs dimensions liées au travail et à l'emploi : les exigences du travail, les exigences émotionnelles, le manque d'autonomie et de marges de manœuvre, les rapports sociaux et relations de travail, les conflits de valeur et enfin l'insécurité socio-économique. S'il existe une riche littérature en épidémiologie sur les liens entre ces risques et différentes pathologies (par exemple, Bonde, 2008 ; Netterstom et al., 2008 ; Kivimäki et al., 2012), leurs déterminants sont moins connus et ont été relativement peu explorés empiriquement (voir Niedhammer et al. (2007) pour quelques caractéristiques des expositions au jobstrain en France ou encore Beque (2014) pour une typologie des expositions en France en 2010).

L'objectif de cet article est d'étudier l'exposition des travailleurs aux risques psychosociaux (RPS) et son évolution entre 2006 et 2010 . La crise économique de 2008 a en effet entraîné une importante dégradation du marché du travail en France (248 000 emplois détruits en 2009), marquée par une forte hausse du taux de chômage (en particulier du chômage de longue durée) et un ralentissement de la progression des salaires (Amar et al., 2011). De par les conséquences de la crise sur l'emploi et les réorganisations du travail, il convient de s'interroger sur une éventuelle dégradation de 1'exposition aux RPS parmi les personnes en emploi (Catalano et Dooley, 1983 ; Fenwick et Tausig, 1994 ; Kieselbach et Triomphe, 2010).

Nous exploitons dans cette optique les deux vagues (2006 et 2010) de l'enquête Santé et itinéraire professionnel (SIP) : elle permet d'interroger les individus en emploi sur leur exposition à différentes dimensions des RPS.

L'article s'organise de la manière suivante. La première partie présente les quinze indicateurs de l'exposition aux RPS retenus et leur évolution entre 2006 et 2010 . La stratégie économétrique est développée dans une deuxième partie.
Les deux parties suivantes présentent les résultats d'estimations relatifs aux déterminants de l'exposition aux RPS et de leur évolution entre 2006 et 2010. Pour expliquer l'évolution observée au cours de cette période, une analyse du rôle de la mobilité professionnelle sur l'exposition aux RPS est proposée ensuite.

\section{Mesure de l'exposition aux RPS et de son évolution entre 2006 et 2010}

$\mathbf{L}$ 9 exposition des travailleurs aux RPS est étudiée à partir de l'enquête Santé et itinéraire professionnel $(S I P)^{1}$ à travers quinze questions présentes dans le questionnaire en 2006 et en 2010 (tableau 1). Ces questions se répartissent selon six dimensions (Collège d'expertise sur le suivi des risques psychosociaux au travail, 2011) : les exigences du travail, les exigences émotionnelles, le manque d'autonomie et de marges de manœuvre, les rapports sociaux et relations de travail, les conflits de valeur et enfin l'insécurité socioéconomique. Pour chacune des quinze questions, l'individu en emploi enquêté doit déclarer la fréquence d'exposition en choisissant l'une des cinq modalités suivantes : « toujours », « souvent », « parfois », « jamais » ou « sans objet ».

La figure I présente l'exposition en 2006 et en 2010 des individus enquêtés occupant un emploi, pour ces quinze RPS. Pour la majorité de ces risques, la proportion d'individus se déclarant exposés augmente de plus de 5 points de pourcentage entre 2006 et 2010. L'augmentation la plus spectaculaire concerne le risque de ne pas avoir un travail reconnu à sa juste valeur : alors que $59 \%$ des individus en emploi se déclaraient exposés en 2006 , ils sont près de $74 \%$ à l'exprimer en 2010. De manière surprenante, seul le fait de déclarer travailler avec la peur de perdre son emploi ne suit pas cette tendance à la hausse malgré le contexte économique défavorable à partir de 2008. Un biais de sélection peut expliquer ce paradoxe apparent. Les individus les plus exposés en 2006 ont une plus grande probabilité de ne plus être en emploi en 2010. Ce biais de sélection n'est pas propre au fait de travailler avec la peur de perdre son emploi et concerne potentiellement l'ensemble des RPS. En effet, en considérant l'exposition aux RPS

1. Cf. Encadré 1 pour une présentation de l'échantillon retenu. 
des individus en emploi, on ne tient pas compte de l'exposition qu'un individu ayant quitté le marché du travail entre 2006 et 2010 aurait potentiellement connue en 2010 s'il participait

\begin{tabular}{|c|c|c|}
\hline \multicolumn{3}{|l|}{$\begin{array}{l}\text { Tableau } 1 \\
\text { Nomenclature des RPS }\end{array}$} \\
\hline Dimension & $\mathrm{N}^{\circ}$ & Intitulé \\
\hline \multirow{5}{*}{ 1. Exigences du travail } & RPS1 & Travailler sous pression \\
\hline & RPS2 & Penser à trop de choses à la fois \\
\hline & RPS3 & Effectuer une quantité de travail excessive \\
\hline & RPS4 & Penser à son travail avant de dormir \\
\hline & RPS5 & Avoir du mal à concilier son travail avec ses obligations familiales \\
\hline \multirow{3}{*}{ 2. Exigences émotionnelles } & RPS6 & Devoir cacher ses émotions ou faire semblant d'être de bonne humeur \\
\hline & RPS7 & Avoir peur pendant son travail \\
\hline & RPS8 & Vivre des tensions avec un public \\
\hline \multirow{2}{*}{ 3. Manque d'autonomie et de marges de manœuvre } & RPS9 & Ne pas pouvoir employer pleinement ses compétences \\
\hline & RPS10 & Avoir très peu de liberté pour décider de la façon de faire son travail \\
\hline \multirow{2}{*}{ 4. Rapports sociaux, relations de travail } & RPS11 & Ne pas avoir un travail reconnu à sa juste valeur \\
\hline & RPS12 & Avoir de mauvaises relations avec ses collègues \\
\hline \multirow{2}{*}{ 5. Conflits de valeurs } & RPS13 & Devoir faire des choses que l'on désapprouve \\
\hline & RPS14 & Ne pas avoir les moyens de faire un travail de qualité \\
\hline 6. Insécurité socio-économique & RPS15 & Travailler avec la peur de perdre son emploi \\
\hline
\end{tabular}

Source : questionnaire de l'enquête SIP $(2006,2010)$ sur les risques psychosociaux.

Figure I

Évolution de l'exposition aux RPS entre 2006 et 2010

(RPS15) Travailler avec la peur de perdre son emploi

(RPS14) Ne pas avoir les moyens de faire un travail de qualité (RPS13) Devoir faire des choses que l'on désapprouve (RPS12) Avoir de mauvaises relations avec ses collègues (RPS11) Ne pas avoir un travail reconnu à sa juste valeur (RPS10) Avoir très peu de liberté pour décider de la façon de faire... (RPS9) Ne pas pouvoir employer pleinement ses compétences (RPS8) Vivre des tensions avec un public

(RPS7) Avoir peur pendant son travail (RPS6) Devoir cacher ses émotions ou faire semblant d'être de.. (RPS5) Avoir du mal à concilier son travail avec ses obligations...

(RPS4) Penser à son travail avant de dormir (RPS3) Effectuer une quantité de travail excessive (RPS2) Penser à trop de choses à la fois (RPS1) Travailler sous pression

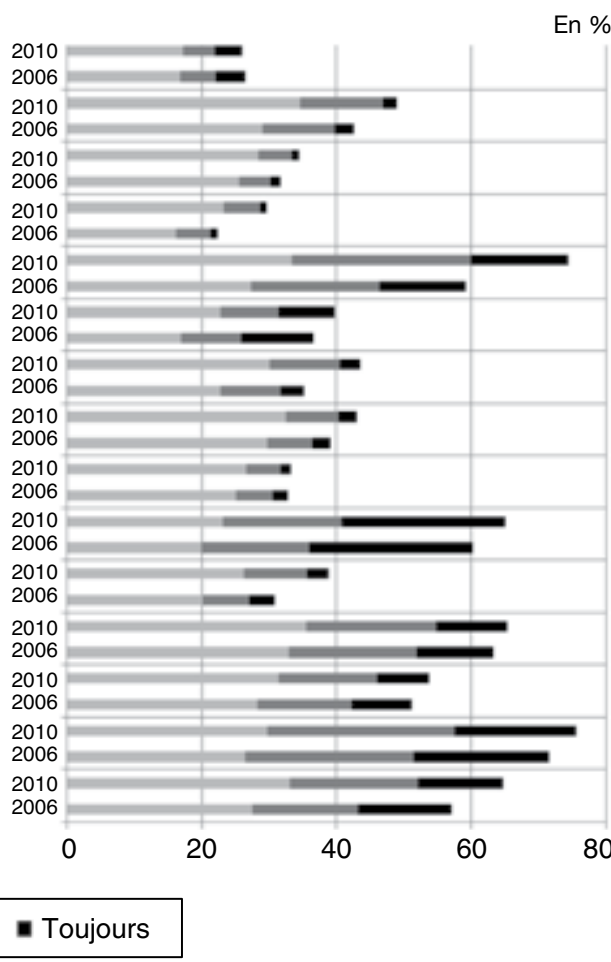

Lecture : en 2006, 28 \% des individus de l'échantillon déclarent parfois travailler sous pression, $15 \%$ souvent travailler sous pression et $14 \%$ toujours travailler sous pression. Au total, $57 \%$ des individus déclarent travailler sous pression en 2006.

Champ : individus âgés de 20 à 68 ans en emploi, déclarant exercer une activité professionnelle en tant que salarié d'une entreprise, d'un artisan, d'une association, d'un particulier, ou en tant que chef d'entreprise ou indépendant, et enquêtés en 2006 ou 2010.

Source : SIP 2006 et 2010 
toujours au marché du travail ${ }^{2}$. Cela conduit a priori à sous-estimer la dégradation de l'exposition aux RPS. Il convient donc de garder à l'esprit que les analyses empiriques menées dans cet article portent sur l'exposition effective aux RPS, conditionnellement au fait de participer au marché du travail et non l'exposition potentielle aux RPS, indépendamment de la participation ou non au marché du travail.

Dans un contexte d'augmentation générale de l'exposition aux RPS, la stabilité de l'exposition au risque de perdre son emploi peut aussi s'expliquer par la manière dont les entreprises françaises se sont ajustées à la crise en accordant une priorité au maintien dans l'emploi des salariés permanents (Cochard et al., 2010). Cette priorité a pu se faire au prix de concessions des salariés sur l'exposition à d'autres RPS. Des mesures de flexibilité interne, de réorganisations du travail liées à la suppression du recours à des salariés temporaires (Perez et Thèvenot, 2014) ou encore des concessions salariales dans le cadre d'accord de sauvegarde de l'emploi ont pu se traduire par une augmentation des exigences, du manque de reconnaissance ou une diminution de l'autonomie au travail.

\section{Stratégie économétrique}

$\mathbf{L}$ exposition aux RPS est mesurée à partir de variables dichotomiques égales à $1 \mathrm{si}$ l'individu déclare être toujours, souvent ou parfois exposé, 0 sinon. Ces variables indiquent si l'individu est exposé ou non à un risque, sans considérer le degré d'exposition. Une des limites de ce choix est que nous ne distinguons pas les individus déclarant être « toujours » exposés de ceux déclarant l'être " parfois », bien que cette information puisse être importante pour comprendre par exemple le lien entre exposition aux RPS et état de santé mentale. Nous précisons dans l'encadré 2 les raisons qui nous ont amenés à retenir ces variables dichotomiques.

Notre analyse empirique est menée en deux temps.

Dans un premier temps, nous estimons par des modèles Probit la probabilité individuelle d'exposition aux différents RPS conditionnellement aux caractéristiques individuelles - caractéristiques socioéconomiques des individus et caractéristiques de leur emploi. Les caractéristiques socioéconomiques retenues dans l'analyse sont le sexe, l'âge, le niveau d'éducation ainsi que la profession et catégorie socioprofessionnelle. Les caractéristiques de l'emploi prises en compte sont le type de contrat (CDD, intérim, $\mathrm{CDI}$ ), le temps de travail, le fait d'exercer ou non un travail posté, un travail de nuit, un travail répétitif, un travail à la chaine et un travail physiquement exigeant.

Les estimations sont effectuées sur l'échantillon des individus en emploi présents à l'une ou l'autre des deux vagues d'enquête. Pour les individus dont l'exposition est mesurée à la fois en 2006 et 2010, il convient de tenir compte d'une probable corrélation dans le temps des résidus. On peut en effet supposer que des facteurs inobservables explicatifs de l'exposition déclarée sont constants dans le temps

2. Ou inversement, l'exposition potentielle en 2006 d'un individu entré sur le marché du travail entre 2006 et 2010.

Encadré 1

\section{ÉCHANTILLON}

L'enquête Santé et itinéraire professionnel (SIP) a été réalisée auprès des ménages ordinaires de France métropolitaine en deux vagues, l'une réalisée à la fin de l'année 2006 et le début de l'année 2007 et l'autre réalisée à la fin de l'année 2010. En 2006-2007, près de 14000 personnes âgées de 20 à 74 ans ont été interrogées. En 2010, la seconde vague a permis de réinterroger 11000 personnes. Pour compléter l'interrogation sur les risques psychosociaux, un échantillon complémentaire d'individus actifs d'au moins 16 ans a été interrogé en 2010 (Coutrot et Mermilliod, 2010 ; Beque, 2014). Le présent article porte sur les personnes âgées de 20 à 68 ans à la date de l'enquête
(2006 pour la première vague, 2010 pour la seconde vague), déclarant exercer une activité professionnelle en tant que salarié d'une entreprise, d'un artisan, d'une association, d'un particulier, ou en tant que chef d'entreprise ou indépendant, au moment de l'une ou l'autre des deux vagues de l'enquête. L'échantillon est ainsi constitué de 3597 travailleurs enquêtés à la fois en 2006 et 2010, 1916 travailleurs enquêtés uniquement en 2006 (ou qui ne sont plus en emploi en 2010) et 2743 travailleurs enquêtés uniquement en 2010 (ou qui n'étaient pas encore en emploi en 2006). Au total, notre échantillon est composé de 8256 individus, soit 11853 individus-années. 
(préférences individuelles de l'individu ou perception subjective des conditions de travail, par exemple). Nous n'intégrons néanmoins pas explicitement ici une composante individuelle inobservable constante dans le temps, mais modélisons la corrélation des résidus qu'elle induit entre 2006 et 2010 en supposant que les résidus du modèle suivent une loi normale bivariée ${ }^{3}$. Par hypothèse, les résidus du modèle sont supposés indépendants des variables explicatives du modèle. Comme évoqué par la suite, cette hypothèse peut être remise en cause dans le cas par exemple de l'étude de l'association entre l'exposition aux RPS et la mobilité professionnelle. Les résultats d'estimation ne doivent donc pas être interprétés en termes d'effets causaux. L'estimation d'un modèle à effets fixes permettrait de ne pas imposer cette hypothèse restrictive d'indépendance des résidus avec les variables explicatives. Cependant, une telle spécification conduit dans le cas d'une variable dépendante qualitative à des estimateurs par maximum de vraisemblance généralement biaisés dès lors que la dimension temporelle est finie, a fortiori quand elle se limite comme dans notre cas à deux périodes ${ }^{4}$ (Greene, 2004 ; Lollivier, 2006). L'observation partielle de la variable latente ne permet pas en effet de réaliser des projections faisant disparaitre le terme individuel (par un estimateur de type « within » par exemple).

Dans un second temps, les résultats d'estimations sont exploités pour étudier l'évolution moyenne de l'exposition aux RPS entre 2006 et 2010 à caractéristiques individuelles observables identiques (voir encadré 3). Il s'agit ainsi de vérifier que l'exposition croissante aux RPS observée sur cette période n'est pas uniquement due à une évolution des caractéristiques individuelles. Pour mettre en évidence des différences d'évolution entre individus au regard de leurs caractéristiques individuelles, les effets marginaux de chacune des caractéristiques individuelles sur l'évolution de l'exposition sont ensuite calculés par une double différence (voir encadré 3). Nous analysons plus particulièrement l'association entre l'évolution de

\footnotetext{
3. Ignorer cette corrélation des résidus conduit à des estimateurs sans biais, mais moins efficaces (Poirier et Ruud, 1988). 4. Sous l'hypothèse que les résidus suivent une loi logistique, une modélisation de type "logit conditionnel " (Chamberlain 1980) permet de définir un modèle à effets fixes. Dans ce cas, l'estimation n'est cependant effectuée que sur l'échantillon des individus dont l'exposition aux RPS varie entre 2006 et 2010. Elle n'a pas été retenue ici car la forte stabilité de l'exposition aux RPS entre 2006 et 2010 implique d'exclure de l'échantillon un nombre très important d'observations.
}

Encadré 2

\section{INDICATEURS DE RPS RETENUS POUR LA MODÉLISATION}

Dans le cadre de cet article, nous avons retenu une approche dichotomique ne tenant pas compte de l'intensité de l'exposition pour les raisons suivantes: les analyses statistiques que nous avons menées montrent que la dégradation quasi-générale de l'exposition aux RPS observée entre 2006 et 2010 et qui, pour l'essentiel, subsiste après contrôle des différences observables entre les travailleurs de 2006 et ceux de 2010, repose sur l'augmentation de la proportion d'individus se déclarant parfois exposés aux RPS (versus ceux déclarant ne jamais être exposés). La probabilité moyenne d'être souvent ou toujours exposé (versus jamais ou parfois) à l'un ou l'autre des RPS ne présente en effet pas d'augmentation claire entre 2006 et 2010 , même après contrôle des caractéristiques observables. Sur les quinze RPS considérés, seuls quatre présentent une augmentation significative de la probabilité moyenne d'être exposé souvent ou toujours. L'augmentation est néanmoins modeste pour trois d'entre elles (+ 1 point de pourcentage) : avoir du mal à concilier sa vie familiale et sa vie professionnelle, ne pas pouvoir employer pleinement ses compétences, ne pas avoir les moyens de faire un travail de qualité. Seul le fait de ne pas avoir un travail reconnu à sa juste valeur présente une forte augmentation
(+ 10 points de pourcentage) après prise en compte d'une définition plus stricte de l'exposition, augmentation que l'on retrouve avec une définition plus souple, comme évoqué précédemment.

D'un point de vue méthodologique, des analyses préliminaires ont montré que l'utilisation d'un modèle multinomial ordonné (permettant une prise en compte plus précise de l'information disponible sur le degré d'exposition via une variable qualitative ordonnée) n'était pas satisfaisante. L'hypothèse d'égalité des pentes est en effet quasiment systématiquement rejetée, indiquant ainsi que l'effet des variables explicatives varie suivant le degré d'exposition considéré. La meilleure approche nous semble-t-il est donc d'étudier dans un premier temps le fait d'être exposé ou non à un RPS et dans un deuxième temps, le degré d'exposition conditionnellement au fait d'être exposé. Menée de manière simultanée, une telle approche s'accompagne cependant d'un certain nombre de défis méthodologiques si l'on souhaite contrôler une partie de l'hétérogénéité inobservée. Dans cet article, nous nous intéressons donc à la première étape et cherchons à expliquer à la fois le risque d'être exposé à un RPS et l'évolution de l'exposition entre 2006 et 2010. 


\section{Encadre 3}

\section{MODÉLISATIONS DE L’EXPOSITION AUX RPS ET DE SON ÉVOLUTION ENTRE 2006 ET 2010}

L'exposition aux RPS est mesurée ici à l'aide des variables dichotomiques $R P S_{k i t}$ égales à 1 si l'individu $i$ déclare être exposé au $k^{\text {ème }}$ RPS $(k=1, \ldots, 15$ à la date $t(t=2006,2010), 0$ sinon.

On définit par ailleurs la variable latente $R P S_{k i t}^{*}$ comme la propension de l'individu $i$ à être exposé au $k^{k^{m}}{ }^{2}$ RPS à la date $t$ :

$R P S_{k i t}=\left\{\begin{array}{l}1 \text { si } R P S_{k i t}^{*} \geq 0 \\ 0 \text { sinon }\end{array}\right.$

L'étude des déterminants de l'exposition aux RPS est réalisée à partir d'un modèle Probit estimé par la méthode du maximum de vraisemblance, avec par hypothèse :

$\operatorname{RPS}_{k i t}^{*}=\alpha_{k t}+X_{i t}^{\prime} \beta_{k t}+u_{k i t}$

Où

- $\alpha_{k t}$ correspond à la constante du modèle, mesurant ainsi la propension à être exposé au $k^{\text {ème }}$ RPS à la date $t$ de l'individu de référence :

- $X_{i t}$ correspond à un vecteur de variables explicatives supposé identique quel que soit le RPS considéré - indépendant de $k$;

- $\beta_{k t}$ correspond à un ensemble de paramètres à estimer et mesure l'effet des variables explicatives sur la propension à être exposé au $k^{\text {ème }}$ RPS à la date $t$;

- $u_{\text {kit }}$ correspond à la composante résiduelle du modèle, supposée indépendante des variables explicatives.

Pour tenir compte de l'existence vraisemblable d'effets individuels inobservables conduisant les termes d'erreur à être corrélés entre 2006 et 2010, les résidus sont supposés suivre une loi normale bivariée pour les individus en emploi présents à la fois en 2006 et 2010 :

$\left(u_{\text {ki2006 }}, u_{\text {ki2010 }}\right) \rightarrow N(\mu, \Omega)$ avec $\mu=\left(\begin{array}{l}0 \\ 0\end{array}\right)$ et $\Omega=\left(\begin{array}{ll}1 & \rho \\ \rho & 1\end{array}\right)$.

Pour les individus présents uniquement en 2006 et 2010 , les résidus sont supposés suivre une loi normale univariée : $u_{k i t} \rightarrow N(0,1)$.

L'approche économétrique mise en œuvre se fixe deux objectifs.

Un premier objectif est d'analyser les déterminants de l'exposition aux RPS. Pour cela, une première spécification du modèle (spécification 1) suppose que les effets des variables explicatives sont identiques en 2006 et 2010 (mais varient d'un RPS à l'autre) : $\beta_{k}=\beta_{k 2006}=\beta_{k 2010}$. Les résultats d'estimation (effets marginaux) sont présentés dans le tableau $A$ en annexe.

Un deuxième objectif est d'analyser les déterminants de l'évolution de l'exposition aux RPS entre 2006 et 2010. L'évolution moyenne est dans un premier temps mesurée toutes choses égales par ailleurs à partir des résultats d'estimation de la spécification 1. Plus précisément, pour chaque individu présent aux deux vagues d'enquête, nous calculons pour chaque RPS la différence entre :

- (i) la probabilité espérée d'exposition aux RPS en 2006:

$\widehat{P}\left(R P S_{k i 2006}=1 \mid X_{i 2006}=x_{i 2006}\right)=F\left(\hat{\alpha}_{k 2006}+x_{i 2006} \hat{\beta}_{k}\right)$

- et (ii) la probabilité espérée d'exposition aux RPS en 2010, en supposant inchangées les caractéristiques individuelles par rapport à 2006 :

$\widehat{P}\left(R S_{k i 2010}=1 \mid X_{i 2010}=x_{i 2006}\right)=F\left(\widehat{\alpha}_{k 2010}+x_{i 2006}{ }^{\prime} \hat{\beta}_{k}\right)$

avec $F$ fonction de répartition de la loi normale centrée réduite.

L'évolution de l'exposition aux RPS entre 2006 et 2010 est dans cette spécification uniquement capturée par la différence au niveau des constantes du modèle, $\alpha_{k 2006}$ et $\alpha_{k 2010}$.

Pour analyser les déterminants de l'évolution de l'exposition aux RPS entre 2006 et 2010, l'hypothèse d'égalité dans le temps des coefficients $\beta_{k t}$ du modèle est relâchée (spécification 2) pour tenir compte d'une possible hétérogénéité des individus au regard de l'évolution des RPS. II est ainsi possible d'étudier, toutes choses égales par ailleurs, les différences d'évolutions d'exposition aux RPS entre hommes et femmes, entre jeunes travailleurs et travailleurs âgés, ou encore selon le niveau d'éducation. Nous mobilisons pour cela une approche en double différence afin de calculer l'effet marginal d'une variable dichotomique sur l'évolution de la probabilité d'être exposé.

Par exemple, pour tester toutes choses égales par ailleurs des différences d'évolution d'exposition aux RPS entre hommes et femmes, on compare pour chaque individu présent en 2006 :

- (i) L'évolution de son exposition espérée conditionnellement au fait qu'il soit un homme (référence) :

$$
\begin{aligned}
& \widehat{P}\left(R P S_{k i 2010}=1 \mid X_{i 2010}^{c}=x_{i 2006}^{c}, \text { femme }_{i 2010}=0\right)- \\
& \widehat{P}\left(R P S_{k i 2006}=1 \mid X_{i 2006}^{c}=x_{i 2006}^{c}, \text { femme }_{i 2006}=0\right)
\end{aligned}
$$


l'exposition au RPS et trois mesures de la crise économique caractérisant cette période.

La première mesure repose sur des indicatrices sectorielles ou de manière alternative sur des agrégats économiques au niveau sectoriel mesurant l'exposition de chaque secteur à la crise (taux de croissance sectoriel de la valeur ajoutée, de l'emploi ou du recours à l'intérim). Une telle approche permet de tester l'hypothèse selon laquelle les individus appartenant aux secteurs d'activité les plus explosés à la crise sont aussi ceux qui ont le plus fréquemment connu une exposition croissante aux RPS.

Une deuxième mesure se situe au niveau de l'entreprise et vise à tester l'existence d'une association significative entre l'évolution de l'exposition aux RPS et l'existence d'un plan de licenciement durant les 12 derniers mois.

Enfin, une troisième mesure appréhende la crise économique de manière plus indirecte à travers les mobilités professionnelles. Elle vise plus précisément à tester l'existence, à caractéristiques individuelles observables données, d'une association significative entre l'évolution de l'exposition aux RPS et le fait d'avoir connu une mobilité professionnelle d'un emploi vers un autre entre 2006 et 2010. Cette approche par les mobilités professionnelles repose sur l'observation, présentée plus en détail dans la dernière partie, d'une réduction de ces mobilités professionnelles en période de crise. Le caractère pro-cyclique de la mobilité professionnelle a été mis en évidence par des données disponibles sur longue période, telles que celles issues de l'enquête Emploi ou celles issues des déclarations mensuelles de mouvements de main-d'œuvre $(D M M O)$ ou des enquêtes trimestrielles sur les mouvements de main-d'œuvre $(E M M O)$. À partir des données de l'enquête SIP, nous étudions l'hypothèse d'une association négative entre mobilité professionnelle et exposition aux RPS, hypothèse pouvant partiellement rendre compte de l'exposition croissante aux RPS observée entre 2006 et 2010. Les données de l'enquête SIP permettent également de distinguer les mobilités internes des mobilités externes et parmi ces dernières, les mobilités volontaires des mobilités subies. La distinction entre mobilités volontaires et mobilités subies s'avère nécessaire car elles peuvent entretenir des relations différentes avec l'évolution de l'exposition aux RPS (voir dernière partie).

Encadre 3 (suite)

(ii), toutes choses égales par ailleurs, l'évolution de son exposition espérée conditionnellement au fait qu'il soit une femme :

$$
\begin{aligned}
& \widehat{P}\left(R P S_{k i 2010}=1 \mid X_{i 2010}^{c}=x_{i 2006}^{c}, \text { femme }_{i 2010}=1\right)- \\
& \widehat{P}\left(R P S_{k i 2006}=1 \mid X_{i 2006}^{c}=x_{i 2006}^{c}, \text { femme }_{i 2006}=1\right)
\end{aligned}
$$

Avec

$\widehat{P}\left(R P S_{k i 2010}=1 \mid X_{i 2010}^{c}=x_{i 2006}^{c}\right.$, femme $\left._{i 2010}=0\right)=$ $F\left(\hat{\alpha}_{k 2010}+x_{i 2006}^{c} \hat{\beta}_{k 2010}^{c}\right)$

$\widehat{P}\left(R P S_{k i 2006}=1 \mid X_{i 2006}^{c}=x_{i 2006}^{c}\right.$, femme $\left._{i 2006}=0\right)=$ $F\left(\hat{\alpha}_{k 2006}+x_{i 2006}^{c} \hat{\beta}_{k 2006}^{c}\right)$

$\widehat{P}\left(R P S_{k i 2010}=1 \mid X_{i 2010}^{c}=x_{i 2006}^{c}\right.$, femme $\left._{i 2006}=1\right)=$ $F\left(\hat{\alpha}_{k 2010}+x_{i 2006}^{c} \hat{\beta}_{k 2010}^{c}+\hat{\beta}_{k 2010}^{\text {femme }}\right)$

$$
\begin{aligned}
& \hat{P}\left(R S_{k i 2006}=1 \mid X_{i 2006}^{c}=x_{i 2006}^{c},\right. \text { femme } \\
& F(2006=1)= \\
& F\left(\hat{\alpha}_{k 2006}+x_{i 2006}^{c}{ }^{\prime} \hat{\beta}_{k 2006}^{c}+\hat{\beta}_{k 2006}^{f e m m e}\right)
\end{aligned}
$$

Où $X_{i t}^{c}$ correspond aux variables explicatives du modèle autres que la variable dichotomique femme $e_{i t}$, $\hat{\beta}_{k t}^{c}$ aux vecteurs de paramètres estimés autre que celui associé à la variable dichotomique femme $e_{i t}$ et $\hat{\beta}_{k t}^{\text {femm }}$ au coefficient estimé associé à la variable dichotomique femme it. $_{\text {. }}$

Comme dans la spécification 1, l'écart entre les constantes de 2006 et 2010 ( $\alpha_{k 2006}$ et $\alpha_{k 2010}$ ) capture l'évolution entre 2006 et 2010 commune à l'ensemble des individus de l'échantillon. La différence entre $\beta_{k 2010}^{f e m m e}$ et $\beta_{k 2006}^{f e m m e}$ capture en plus une évolution spécifique aux femmes. La même procédure est utilisée pour l'ensemble des variables explicatives du modèle. Les évolutions moyennes associées à chaque variable explicative sont présentées dans le tableau $B$ en annexe. Cette méthode est également utilisée pour évaluer l'effet sur l'évolution de l'exposition aux RPS entre 2006 et 2010 du secteur d'activité, de l'existence d'un plan de licenciement et du fait d'avoir connu une mobilité professionnelle. 
Toutefois, l'estimation de l'effet des mobilités professionnelles sur l'exposition aux RPS peut être affectée par un biais d'endogénéité. On peut en effet raisonnablement supposer l'existence d'une causalité inverse, au sens où les mobilités professionnelles peuvent dépendre de l'évolution (réelle ou souhaitée) de l'exposition aux RPS. Théoriquement, l'endogénéité de la mobilité professionnelle peut être prise en compte par une méthode d'instrumentation mais la mise en œuvre d'une telle méthode nécessite d'identifier des instruments exclus valides, ce qui s'avère difficile en l'espèce. L'analyse du lien entre mobilité professionnelle et exposition aux RPS proposée dans cet article ne repose donc pas sur une analyse causale mais sur une analyse en termes de corrélation.

\section{Déterminants socioéconomiques de l'exposition aux RPS}

L'

exposition aux RPS diffère selon le genre. Les femmes sont, toutes choses égales par ailleurs, significativement plus exposées que les hommes aux exigences du travail, au fait de devoir cacher leurs émotions ou de faire semblant d'être de bonne humeur et à l'insécurité socioéconomique (voir les résultats de la spécification 1 dans le tableau A en annexe). Elles sont en revanche moins exposées que les hommes au risque d'avoir peur pendant leur travail et au risque de faire des choses qu'elles désapprouvent. Les coefficients relatifs à l'âge présentent un effet « en U inversé ». Les 35-44 ans apparaissent d'une manière générale comme les plus exposés aux RPS. À partir de 45 ans, l'exposition aux RPS diminue en effet avec l'âge pour une majorité de RPS. Les moins de 35 ans sont moins exposés que les 35-44 ans aux exigences du travail et à l'insécurité socioéconomique mais sont plus exposés au manque d'autonomie et de marges de manœuvre. Les coefficients relatifs au diplôme ${ }^{5}$ présentent également un effet « en U inversé ». Les travailleurs sans diplôme ou de niveau BEPC et ceux de niveau CAP ou BEP sont moins exposés que ceux ayant un niveau baccalauréat pour tous les items sauf deux, la «peur pendant le travail» et «travailler avec la peur de perdre son emploi ». Les travailleurs de niveau bac +2 ou plus sont quant à eux moins exposés que les travailleurs de niveau baccalauréat, même si le nombre d'items pour lesquels l'effet est significatif est plus faible.

Les profils d'exposition aux RPS divergent également entre individus selon leur catégorie professionnelle. Les cadres et professions intellectuelles, les professions intermédiaires et dans une moindre mesure les artisans, commerçants et chefs d'entreprise sont plus exposés aux exigences du travail, aux exigences émotionnelles et aux conflits de valeur, par comparaison aux employés. Ils sont en revanche moins exposés au manque d'autonomie et de marges de manœuvre. Toutes choses égales par ailleurs, en particulier après contrôle des caractéristiques de l'emploi, les ouvriers apparaissent significativement moins exposés que les employés sur l'ensemble des RPS, mais ils sont plus exposés au « manque de liberté pour décider de la façon de faire leur travail» et à la «peur pendant le travail ». Ces résultats rejoignent ceux mis en évidence par d'autres études (voir par exemple Bué et al., 2008). Enfin, comparativement aux employés, les agriculteurs apparaissent quant à eux surexposés sur toutes les dimensions à l'exception de l'insécurité socioéconomique et des exigences émotionnelles.

Les caractéristiques de l'emploi jouent un rôle prépondérant pour rendre compte de l'exposition des travailleurs aux RPS. Disposer d'un contrat à durée limitée (i.e. un contrat à durée déterminée ou un contrat d'intérim) augmente de manière attendue l'insécurité socioéconomique et le manque d'autonomie et de marge de manœuvre. En revanche, cela s'accompagne d'une moindre exposition aux exigences du travail, aux mauvaises relations de travail et aux conflits de valeurs. Le fait de ne pas envisager sa carrière dans l'emploi actuel pourrait ainsi rendre plus supportable ces mauvaises relations ou ces conflits. Les travailleurs indépendants ${ }^{6}$ ne se distinguent pas des salariés au regard de l'exposition aux exigences émotionnelles et de l'insécurité socioéconomique. Ils sont en revanche protégés sur l'ensemble des autres dimensions.

La durée hebdomadaire de travail apparait être un facteur de surexposition aux RPS. Déclarer travailler fréquemment plus de 48 heures par semaine est corrélé aux exigences du travail, aux exigences émotionnelles, aux difficultés dans les rapports sociaux et relations de travail

5. Pour ne pas supprimer de l'échantillon les individus pour lesquels l'information est manquante sur le diplôme et la PCS, en proportion non négligeable, nous avons créé pour chacune des deux variables une modalité "non réponse ».

6. Afin d'explorer plus finement les différences entre salariés et indépendants, nous avons considéré une modélisation plus souple, consistant à croiser chaque caractéristique individuelle avec l'indicatrice « indépendant ». Les différences sont rarement significatives. Dans le prolongement de ce travail, une analyse plus détaillée de la population des indépendants, tenant compte de la diversité des métiers, permettrait de mettre en évidence les spécificités de cette population en matière d'exposition aux RPS. 
et à l'insécurité socioéconomique. De plus, les différentes dimensions des RPS sont toutes associées aux indicatrices de pénibilités physiques introduites dans les régressions. Exercer un travail posté, un travail de nuit, un travail à la chaine ou répétitif ou encore un travail physiquement exigeant sont, pour une majorité de RPS, significativement associés à la probabilité d'exposition. Ce résultat souligne l'existence d'un phénomène de cumul des pénibilités physiques et psychosociales, qui peut avoir des conséquences particulièrement nuisibles pour la santé physique et mentale.

Enfin, les régressions mettent en évidence des différences d'exposition aux RPS entre secteurs d'activité. À partir des effets marginaux associés à chaque secteur, nous avons construit des indices synthétiques permettant de classer les secteurs d'activité en fonction du degré d'exposition moyen des individus y travaillant ${ }^{8}$. Les résultats détaillés ne sont pas présentés dans cet article mais sont disponibles sur demande.

Une opposition entre l'industrie et les services apparaît dans la nature des expositions. Les difficultés dans les rapports sociaux et les relations au travail sont particulièrement prégnantes dans plusieurs secteurs de l'industrie - automobile, industries agroalimentaires, industries du bois, du papier, du caoutchouc et des plastiques, production et distribution d'électricité, eau, gaz et chaleur. Dans ces industries, plus exposées à la concurrence internationale et confrontées à des difficultés économiques entraînant des restructurations, les travailleurs ont ainsi plus souvent un sentiment de faible reconnaissance au travail et les relations entre collègues se révèlent être moins bonnes que dans les autres secteurs. Les individus travaillant dans les services sont beaucoup moins exposées à ces difficultés. Ils apparaissent en revanche davantage confrontés aux exigences émotionnelles. Les secteurs les plus exposés sont les transports, l'éducation, la santé, l'action sociale, l'immobilier et l'assainissement, voirie et gestion des déchets. Dans ces dernières, les confrontations avec un public, impliquant de devoir cacher ses émotions, et le sentiment de peur pendant le travail peuvent expliquer ce niveau élevé d'exposition. Plusieurs secteurs des services sont également particulièrement exposés aux conflits de valeurs, i.e. devoir faire des choses que l'on désapprouve et ne pas avoir les moyens de faire un travail de qualité.

Certains secteurs de l'industrie - la production de pétrole et de gaz, la distribution d'électricité, eau, gaz et chaleur - et la construction sont particulièrement concernés par les exigences au travail. Le manque d'autonomie et de marge de manœuvre apparaît tout particulièrement dans l'automobile et la cokéfaction et le raffinage. Enfin, le sentiment d'insécurité socioéconomique est le plus souvent déclaré dans les industries textiles, la métallurgie, la poste et les télécommunications et les activités informatiques, qui ont connu des réductions d'effectifs, mais également dans l'assainissement, la voirie et gestion des déchets.

$\mathrm{Au}$ total, ces estimations mettent en évidence, d'une part, une surexposition aux RPS pour les individus en emploi âgés de 35 à 44 ans, ceux ayant un diplôme de niveau baccalauréat, ainsi que ceux exposés à des pénibilités physiques (travail physiquement exigeant, répétitif ou à la chaîne, travail posté ou de nuit). Ces populations devraient constituer des cibles prioritaires en termes de politique de prévention des RPS et d'amélioration des conditions de travail. D'autre part, elles mettent en lumière la nécessité de distinguer les différentes dimensions des RPS pour en comprendre les déterminants, avec des profils d'exposition différenciés selon le genre, la catégorie professionnelle et le secteur d'activité.

Au-delà de ces déterminants socioéconomiques observables, l'estimation des coefficients de corrélation entre les résidus des équations de 2006 et 2010 montre que des déterminants inobservables expliquent conjointement les expositions en 2006 et 2010. Les coefficients de corrélation des résidus estimés sont en effet tous significativement positifs et relativement élevés, allant de 0,33 (pour la variable RPS1 : travailler sous pression) à 0,56 (pour la variable RPS 4 : penser à son travail avant de s'endormir). Une forte stabilité dans le temps existe donc dans l'exposition aux RPS. La figure II illustre ce résultat: être exposé aux RPS en 2006 accroit de manière importante le risque d'être exposé aux RPS en 2010, à caractéristiques observables données.

7. L'enquête SIP nous a permis de distinguer 30 secteurs d'activité différents auxquels se rattachent les emplois des enquêtés.

8. Pour chacun des quinze RPS, nous avons dans un premier temps caractérisé chaque secteur par son rang au regard de la valeur de l'effet marginal qui lui est associé (du moins exposé au plus exposé). Dans un second temps, nous avons construit des indices synthétiques par dimension en sommant pour chaque secteur les rangs des RPS d'une même dimension. 


\section{Déterminants de l'aggravation de l'exposition entre 2006 et 2010}

\author{
Évolution moyenne \\ et selon les caractéristiques individuelles
}

Afin d'étudier si l'augmentation de l'exposition mise en évidence dans la figure I provient d'un effet de structure, nous avons au sein de l'échantillon de 2006, et pour chaque item, comparé la probabilité moyenne d'exposition en 2006 avec la probabilité prédite moyenne d'être exposé en 2010 en supposant inchangées les caractéristiques individuelles. Les augmentations sont moins nettes une fois que l'on tient compte des différences de caractéristiques entre les travailleurs de 2006 et ceux de 2010 (figure III). Pour près de la moitié des RPS, on constate néanmoins une exposition significativement plus fréquente en 2010, signe d'une aggravation de l'exposition sur la période 2006-2010 indépendante des changements de caractéristiques individuelles.

Qui sont les travailleurs caractérisés par une augmentation de l'exposition aux RPS ? Pour répondre à cette question nous avons calculé les effets marginaux des caractéristiques individuelles sur l'évolution de l'exposition aux RPS entre 2006 et 2010, à partir de la spécification 2 (voir encadré 3). Les résultats sont présentés dans le tableau $\mathrm{B}$ en annexe.

Les résultats d'estimation indiquent une évolution de l'exposition aux RPS différenciée selon l'âge des enquêtés. Le fait d'appartenir aux classes d'âge 45-54 ans ou à celle des 55 ans et plus a eu un effet protecteur vis-à-vis de l'évolution du risque d'être exposé à certaines demandes ou exigences: les estimations indiquent un moindre risque d'avoir trop de choses à penser à la fois, de devoir cacher ses émotions, d'avoir à effectuer une quantité excessive de travail (uniquement pour les 55 ans et plus) et d'avoir peur pendant son travail (uniquement pour les 45-54 ans), par comparaison aux enquêtés âgés de 35 à 44 ans. De plus, les travailleurs les plus jeunes (moins de 35 ans) ont vu croître leur risque d'avoir très peu de liberté pour décider de la façon de faire leur travail. Ainsi, les ajustements du travail pendant la période de crise ont davantage concerné la main d'œuvre âgée de moins de 44 ans. Cette main

Figure II

Probabilités prédites moyennes d'être exposé aux RPS en 2010 conditionnellement à l'exposition de 2006

(RPS15) Travailler avec la peur de perdre son emploi

(RPS14) Ne pas avoir les moyens de faire un travail de qualité (RPS13) Devoir faire des choses que l'on désapprouve (RPS12) Avoir de mauvaises relations avec ses collègues (RPS11) Ne pas avoir un travail reconnu à sa juste valeur (RPS10) Avoir très peu de liberté pour décider de la façon de faire...

(RPS9) Ne pas pouvoir employer pleinement ses compétences (RPS8) Vivre des tensions avec un public (RPS7) Avoir peur pendant son travail (RPS6) Devoir cacher ses émotions ou faire semblant d'être de... (RPS5) Avoir du mal à concilier son travail avec ses obligations... (RPS4) Penser à son travail avant de dormir (RPS3) Effectuer une quantité de travail excessive (RPS2) Penser à trop de choses à la fois (RPS1) Travailler sous pression

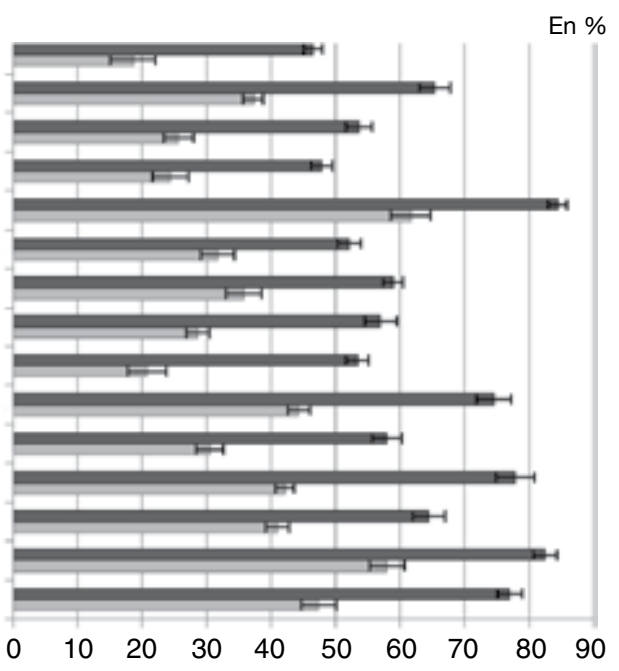

- Sachant exposé en 2006

Sachant non exposé en 2006

Note : ces probabilités ont été prédites à partir des résultats d'estimation de la spécification 1 du modèle. Les moyennes présentées dans le graphique ont été calculées sur les individus enquêtés en 2006 et 2010 (individus du panel).

Lecture : conditionnellement au fait de ne pas être exposé en 2006, la probabilité d'être exposé en 2010 au RPS1 (Travailler sous pression) est en moyenne égale à $47 \%$. Conditionnellement au fait d'être exposé en 2006, cette même probabilité est en moyenne égale à $77 \%$. Les barres d'erreur représentent les intervalles de confiance à $95 \%$.

Champ : enquêtés âgés de 20 à 68 ans en emploi, déclarant exercer une activité professionnelle en tant que salarié d'une entreprise, d'un artisan, d'une association, d'un particulier, ou en tant que chef d'entreprise ou indépendant, enquêtés en 2006 et 2010.

Source : SIP 2006 et 2010 
d'œuvre plus jeune peut en effet être davantage sujette à ces ajustements du fait d'une moindre protection de l'emploi (liée notamment à une moindre ancienneté dans l'entreprise ou une représentation syndicale moins forte); cette moindre protection de l'emploi apparaît dans nos estimations: les individus de moins de 45 ans sont plus exposés à la crainte de perdre leur emploi par comparaison aux travailleurs de 45 ans et plus (voir spécification 1, tableau A en annexe). Les femmes ne semblent pas avoir vu leur situation se dégrader davantage que celles des hommes hormis pour l'item 13 « devoir faire des choses que l'on désapprouve ». Le niveau de diplôme a joué sur le manque d'autonomie qui a davantage augmenté pour l'item « ne pas pouvoir employer pleinement ses compétence » à la fois pour les niveaux CAP, BEP et bac +2 ou plus, par rapport au niveau baccalauréat. Les cadres et les ouvriers ont vu leur situation se dégrader du point de vue des exigences du travail relativement aux employés. Le travail à durée limitée a eu un effet protecteur contre l'augmentation de l'exposition selon plusieurs items, sans doute du fait des mobilités professionnelles plus fréquentes (cf. dernière partie). Enfin, le travail répétitif ou à la chaîne a encore accru le manque d'autonomie ainsi que celui d'avoir une quantité excessive de travail.

Ainsi, à l'exception de l'âge, peu de caractéristiques individuelles sont significativement associées à l'évolution de l'exposition aux RPS et quand elles le sont, elles sont spécifiques à certains RPS. Ces résultats montrent que l'accroissement général de l'exposition observé sur la période 2006-2010 n'est pas propre à une catégorie de travailleurs.

\section{Une évolution liée à la crise?}

Dans quelle mesure l'exposition aux RPS dépend-elle du contexte économique? Pour répondre à cette question, la littérature empirique sur le lien entre les périodes de récession économique, les conditions de travail et la santé des travailleurs distingue une relation de court terme et une relation de moyen terme. Sur le court terme (i.e. au cours de l'année de la récession), le ralentissement économique peut entraîner une surcapacité temporaire des entreprises et une diminution de la charge de travail. L'exposition des travailleurs aux RPS peut alors

\section{Figure III \\ Probabilités prédites moyennes d'être exposé aux RPS en 2006 et 2010, après contrôle des caractéristiques individuelles}

(RPS15) Travailler avec la peur de perdre son emploi

(RPS14) Ne pas avoir les moyens de faire un travail de qualité

(RPS13) Devoir faire des choses que l'on désapprouve

(RPS12) Avoir de mauvaises relations avec ses collègues

(RPS11) Ne pas avoir un travail reconnu à sa juste valeur

(RPS10) Avoir très peu de liberté pour décider de la façon de faire...

(RPS9) Ne pas pouvoir employer pleinement ses compétences

(RPS8) Vivre des tensions avec un public

(RPS7) Avoir peur pendant son travail

(RPS6) Devoir cacher ses émotions ou faire semblant d'être de...

(RPS5) Avoir du mal à concilier son travail avec ses obligations...

(RPS4) Penser à son travail avant de dormir

(RPS3) Effectuer une quantité de travail excessive

(RPS2) Penser à trop de choses à la fois (RPS1) Travailler sous pression

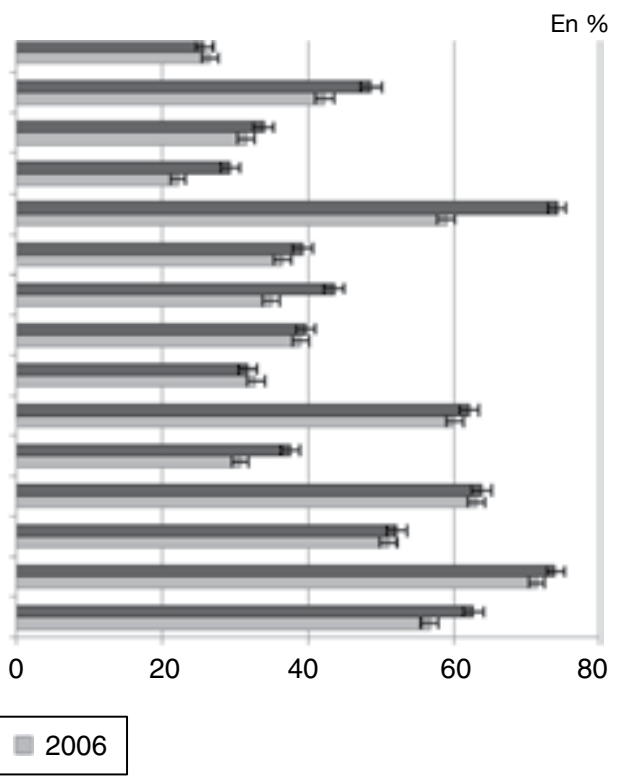

Note : ces probabilités ont été prédites à partir des résultats d'estimation de la spécification 1 du modèle (cf. encadré 3). Les moyennes présentées dans le graphique ont été calculées sur les individus enquêtés en 2006.

Lecture : en moyenne, les individus de l'échantillon ont une probabilité égale à $57 \%$ d'être exposés au RPS 1 (Travailler sous pression) en 2006. À caractéristiques individuelles inchangées, la probabilité moyenne prédite en 2010 est égale à $63 \%$, soit une augmentation significative de 6 points de pourcentage. Les barres d'erreur représentent les intervalles de confiance à $95 \%$.

Champ : individus âgés de 20 à 68 ans en emploi, déclarant exercer une activité professionnelle en tant que salarié d'une entreprise, d'un artisan, d'une association, d'un particulier, ou en tant que chef d'entreprise ou indépendant, enquêtés en 2006 ou 2010.

Source : SIP 2006 et 2010. 
diminuer momentanément et leur état de santé s'en trouver amélioré. Néanmoins, à moyen terme (i.e. au cours des trois années qui suivent l'année marquée par la récession), les ajustements de l'emploi à la baisse peuvent accroître la pression sur les travailleurs qui restent en emploi et réduire leurs marges de manœuvre. Pour les travailleurs qui conservent leur emploi après une vague de réduction d'effectif, il y a un risque d'aggravation de l'exposition aux RPS. Il s'agit là du " syndrome du survivant » (Noer, 1997 ; Kieselbach et Triomphe, 2010) ; les « rescapés » peuvent alors être tout particulièrement concernés par un sentiment d'incertitude à l'égard de l'évolution de l'entreprise et de leur emploi. Ce dernier peut se cumuler avec une augmentation de leur charge de travail.

Les résultats indiquent sur le court terme une amélioration des indicateurs de santé physique, une diminution des accidents du travail et de la mortalité en période de récession (Ruhm, 2003, 2004 ; Buchmueller et al., 2006 ; Robinson et Shor, 1989; Yahou et Bouvet, 2001 ; Ussif, 2004 ; Davies et al., 2009 ; Asfaw et al., 2011). Toutefois, plusieurs travaux soulignent que la baisse constatée des accidents du travail peut être davantage imputable à une accentuation de la sous-déclaration des accidents bénins qu'à une baisse des risques d'accidents (Boone et van Ours, 2006). De plus, ces travaux constatent une dégradation de l'état de santé mentale en période de récession (Ruhm, 2003 ; Charles et DeCicca, 2008 ; et McInerney et Mellon, 2012). Sur le moyen terme, les périodes de récessions s'accompagnent, de façon non ambigüe, d'une dégradation des conditions de travail et de l'état de la santé physique et mentale (Catalano et Dooley, 1983 ; Brenner, 1987 ; Fenwick et Tausig, 1994 ; Coile, 2012 ; Bender et al., 2013). Par ailleurs, une littérature empirique abondante en sciences sociales (Ferrie et al., 1998, 2005, 2007; Kieselbach et Triomphe, 2010) fait état d'une dégradation de l'état de santé des travailleurs restant dans l'entreprise suite à des réductions d'effectif, ainsi qu'une augmentation de leur consommation de médicaments psychotropes et une consommation accrue de tabac et d'alcool.

Bien que la littérature tende à démontrer une association significative entre le contexte macroéconomique, les conditions de travail et la santé des travailleurs, il est difficile d'identifier avec seulement deux points d'observation si la crise de 2008 est à l'origine de l'aggravation générale de l'exposition aux RPS observée entre 2006 et 2010 . Il est impossible, sans hypothèses paramétriques fortes, de dissocier rigoureusement ce qui s'explique par une tendance de fond (préalable à l'apparition de la crise) de ce qui est directement imputable à la crise. Une telle évaluation nécessiterait de disposer d'au moins un point d'observation supplémentaire avant la crise.

A minima, il est néanmoins possible d'évaluer dans quelle mesure l'évolution de l'exposition aux RPS est associée à l'évolution du contexte macroéconomique sur la période 2006-2010. Nous avons examiné l'hypothèse selon laquelle les individus appartenant à des secteurs d'activité ayant connu un choc d'activité négatif important ont subi une plus forte aggravation de leur exposition aux RPS. Les résultats d'estimation relatifs aux indicatrices de secteur introduites dans la spécification 2 (non présentées dans l'article) ne permettent pas de valider cette hypothèse. Les effets marginaux associés à ces indicatrices tendent en effet à montrer que, toutes choses égales par ailleurs, les différences sectorielles d'évolution de l'exposition aux RPS sont très peu marquées et très rarement significatives sur la période 2006-2010. Autrement dit, malgré l'hétérogénéité des secteurs au regard de l'exposition à la crise, quasiment aucune différence significative n'est à noter dans l'évolution de l'exposition aux RPS par secteur d'activité. Ce résultat est confirmé par le calcul de coefficients de corrélation entre les effets marginaux associés aux indicatrices de secteur et différents agrégats économiques mesurés au niveau du secteur : les évolutions apparaissent indépendantes du taux de croissance sectoriel de la valeur ajoutée, de l'emploi ou encore du taux de recours à l'intérim, ce dernier étant a priori un bon indicateur d'exposition à la crise.

Cette absence de relation significative peut s'expliquer par un manque de puissance statistique (certains secteurs d'activité étant peu représentés dans l'échantillon) ou par un biais de déclaration lié aux secteurs d'appartenance des individus enquêtés ${ }^{9}$. Elle peut aussi révéler le fait que l'évolution de l'exposition aux RPS se joue à un niveau plus local que ne peut mesurer un agrégat au niveau du secteur d'activité.

Le questionnaire SIP de la vague 2010 permet d'identifier les individus appartenant à une entreprise ayant fait face à un plan de

9. On note une fréquence particulièrement élevée de changement de secteur entre 2006 et 2010, sans changement d'employeur, qui laisse suspecter des biais dans la déclaration du secteur. 
licenciement durant les 12 derniers mois. Parmi les individus salariés enquêtés en 2010 , près de $13 \%$ déclarent que leur entreprise a connu un plan de licenciement. L'ajout de cette information dans la spécification 2 du modèle montre que l'existence d'un plan de licenciement tend à augmenter sur la période 2006-2010 l'exposition aux RPS toutes choses égales par ailleurs. L'effet est significatif au seuil de $5 \%$ pour 3 items : « Ne pas pouvoir employer pleinement ces compétences» $(+0,07$ point de pourcentage), «penser à son travail avant de s'endormir» $(+0,05$ point de pourcentage $)$ et « travailler avec la peur de perdre son emploi » $(+0.07$ point de pourcentage).

Le fait que l'évolution de l'exposition aux RPS ne soit pas significativement associée aux agrégats économiques mesurés (taux de croissance de la valeur ajoutée, de l'emploi ou de recours à l'intérim) au niveau des secteurs d'activité n'exclut donc pas que la dégradation de la conjoncture ait un effet à un niveau plus désagrégé. L'analyse au niveau individuel du lien entre l'évolution de l'exposition aux RPS et la mobilité professionnelle moins fréquente en période de crise, présentée dans la section suivante étaye ce résultat.

\section{Une moindre mobilité professionnelle en période de crise est-elle source d'aggravation de l'exposition aux RPS ?}

\section{Le caractère pro-cyclique de la mobilité} professionnelle

Si les récessions provoquent des destructions d'emploi, elles semblent s'accompagner également d'une réduction de la mobilité sur le marché du travail. Amossé (2003) montre le caractère pro-cyclique de la mobilité externe pendant les années 1990. Les perspectives économiques des entreprises se dégradant, elles sont moins enclines à embaucher et les salariés, de leur côté, feraient preuve d'une plus grande prudence dans leur décisions de mobilité durant les périodes de ralentissement de l'activité. Ce lien entre mobilité et cycle économique est confirmé par Lalé (2010) qui met en lumière sur la période 1982-2007 à partir des données de l'enquête Emploi une corrélation négative entre taux de chômage et mobilité.

Qu'en est-il pour la crise de 2008? Les déclarations mensuelles de mouvements de main-d'œuvre (DMMO) et les enquêtes trimestrielles sur les mouvements de main-d'œuvre $(E M M O)$ réalisées ces 15 dernières années confirment la réduction de la mobilité professionnelle externe pour la grande majorité des travailleurs. Les différents motifs d'entrée et sortie qui sont distingués dans ces sources permettent d'analyser la mobilité des salariés en CDD et celles des salariés en CDI.

Dans le processus d'ajustement de l'emploi à l'activité en 2008 , la priorité a été donnée à la réduction des flux d'entrée en CDI dans les établissements. Les flux de sortie de CDI ont eux aussi suivi ce mouvement à la baisse ${ }^{10}$. Les salariés en CDI qui représentent près de $90 \%$ des salariés du secteur privé ont donc connu une réduction de leur mobilité (Dares, 2015). Les mouvements des salariés en CDD se caractérisent par une légère tendance à la hausse. Comme pour les CDI, les mouvements ont diminué début 2008, mais ils ont ensuite connu une augmentation qui s'est accentué en 2009 et 2010 (Dares, 2015). On peut donc conclure que malgré une augmentation temporaire des licenciements économiques, la mobilité externe a diminué pour une très large majorité de salariés, ceux titulaires d'un CDI. Les entreprises ont en effet adopté un comportement de rétention de leurs salariés en CDI en mobilisant différents mécanismes de flexibilité interne: recours aux heures supplémentaires, recours au chômage partiel (Cochard et al., 2010 ; Ananian et al., 2012). Cela s'est traduit par une baisse de l'emploi moins importante qu'attendu, compte tenu de la chute de l'activité (Argouarc'h et al., 2010). Une grande partie de l'ajustement a porté sur l'emploi périphérique (intérim, CDD). L'emploi intérimaire a en particulier joué le rôle d'amortisseur. Alors qu'il ne représentait dans l'industrie que 3,6\% des effectifs salariés avant la crise, les destructions d'emplois qui ont eu lieu entre le $1^{\text {er }}$ avril 2008 et le 30 juin 2009 concernaient des emplois intérimaires à hauteur de $53 \%$ (Centre d'analyse stratégique, 2009). Dans le secteur des services marchands, ce sont les CDD qui ont majoritairement servi d'amortisseur de choc d'activité.

\section{Quel lien entre mobilité professionnelle et exposition aux RPS?}

La littérature empirique montre que les individus les plus exposés aux RPS seraient plus enclins à la mobilité professionnelle dans un

\footnotetext{
10. Seules les ruptures conventionnelles qui font leur apparition début 2008 et les licenciements économiques (sur trois trimestres seulement: $4^{e}$ trimestre 2008-2 $2^{e}$ trimestre 2009) connaissent une augmentation, mais ces mouvements sont plus que compensés par la baisse des démissions, des licenciements pour autres motifs et de rupture pendant la période d'essai.
} 
avenir proche. Selon Amossé et Gollac (2008), à partir d'une exploitation de l'enquête française « formation et qualification professionnelle » de 2003, une forte intensité du travail est associée à une probabilité accrue de mobilité. Un travail sur données suédoises basé sur une approche des RPS fondée sur les modèles demande/ contrôle (Karasek et Theorell, 1990) et efforts/ récompenses (Siegrist, 1996) conduit au même type de résultats sur le lien entre exposition initiale et mobilité (Söderberg et al., 2014). Ces travaux se focalisent sur l'exposition avant la mobilité. L'enquête SIP nous permet d'observer l'exposition avant et après la mobilité et de calculer son évolution afin d'étudier l'hypothèse d'une mobilité réduisant l'exposition. Si cette hypothèse est corroborée, et si l'on s'appuie sur le constat d'une baisse de la mobilité pendant la crise, la dégradation de l'exposition aux RPS observée entre 2006 et 2010 pourrait en partie s'expliquer par la moindre mobilité professionnelle caractérisant cette période.

Le lien entre mobilité professionnelle et exposition aux RPS est ici analysé en se focalisant sur les mobilités professionnelles d'un emploi vers un autre ${ }^{11}$. Trois types de mobilités sont distingués : la mobilité externe volontaire (changement d'employeur suite à une démission, un refus par le salarié de renouveler un contrat ou une rupture conventionnelle), la mobilité externe subie (changement d'employeur suite à une fin de contrat ou de mission ou à un non renouvellement par l'employeur ou licenciement) et la mobilité interne (changement de profession, de fonctions ou de lieu géographique sans changement d'employeur).

Mobilité externe volontaire et mobilité externe subie nécessitent d'être distinguées dans la mesure où elles ont connu une trajectoire différente sur la période considérée. Ces différents types de mobilité peuvent en outre entretenir des liens différents avec l'évolution de l'exposition aux RPS. On peut en particulier faire l'hypothèse que la mobilité externe volontaire s'accompagne d'une réduction de l'exposition aux RPS, cette dernière pouvant être un des objectifs de la mobilité. Si la volonté de bénéficier d'une meilleure rémunération est le premier moteur d'une demande de mobilité à l'initiative du salarié, la volonté d'avoir de meilleures conditions de travail apparait en effet comme la deuxième motivation (enquête TNS, Conseil d'orientation pour l'emploi, 2009). Selon cette même enquête, lorsque la mobilité est proposée par 1'entreprise, $35 \%$ des salariés considèrent un accroissement de l'autonomie comme un atout important du nouveau poste.

Le lien entre mobilité subie et évolution de l'exposition est a priori plus ambigu. La mobilité n'étant pas à l'initiative du salarié, le lien avec l'évolution de l'exposition aux RPS est sans doute plus distendu. Dans la mesure où la mobilité a été subie, on peut penser qu'elle n'a pas pu être aussi bien préparée qu'une mobilité volontaire et qu'elle n'a pas permis au salarié d'attendre de trouver un emploi moins exposé pour changer d'employeur. Néanmoins, même en cas de mobilités subies, la réduction de l'exposition aux RPS peut faire partie des objectifs des salariés dans leur recherche d'un nouvel emploi. Parmi eux, les salariés qui craignaient en 2006 de perdre leur emploi, et qui l'ont effectivement perdu, sont sans doute moins exposés à cette peur dans leur nouvel emploi en 2010. Cette réduction de l'exposition semble aussi s'observer sur d'autres RPS. Selon l'enquête de la TNS Sofres menée à l'initiative du Conseil d'orientation pour l'emploi, $41 \%$ des salariés confrontés à une mobilité subie mettent en avant de meilleures conditions de travail dans leur nouvel emploi et $33 \%$ une meilleure conciliation entre vie privée et vie professionnelle.

Les données de l'enquête SIP permettent d'appréhender plus précisément le lien entre mobilité professionnelle et exposition aux RPS, les individus étant interrogés en 2010 sur leurs éventuelles mobilités professionnelles depuis 2006. Sur la période 2006-2010, la mobilité externe volontaire caractérise $9 \%$ de notre échantillon de travailleurs. La mobilité externe subie caractérise $6 \%$ de l'échantillon sur la période $2006-2010$ et $21 \%$ des travailleurs disposant d'un contrat de travail avec limitation de durée. Enfin la mobilité interne caractérise $23 \%$ des individus de notre échantillon sur la période 2006-2010, sans qu'il soit possible au regard des informations disponibles de distinguer mobilité volontaire ou subie.

\section{Résultats d'estimation}

Pour évaluer empiriquement les liens entre ces différentes mobilités et l'évolution des RPS, des variables indicatrices des mobilités professionnelles caractérisant la période 2006-2010 sont ajoutées à la spécification 2 du modèle (voir encadré 3). Les résultats d'estimations sont

11. Les mobilités de l'emploi vers le chômage ou du chômage vers l'emploi ne sont pas étudiées car notre analyse porte sur l'évolution de l'exposition aux RPS des individus en emploi. 
ensuite utilisés pour prédire pour chaque individu de l'échantillon l'exposition espérée aux RPS en 2006 et 2010, avec et sans mobilité professionnelle, en contrôlant des caractéristiques individuelles observables.

Les prédictions montrent qu'il existe une forte association entre mobilité professionnelle et exposition aux RPS. Avant de connaitre une mobilité professionnelle, les individus tendent d'une manière générale à être caractérisés par une plus forte exposition aux RPS. Sans tenir compte du type de mobilité professionnelle, la probabilité d'être exposé en 2006 est en effet toujours supérieure chez les individus qui connaitront une mobilité dans les 4 années suivantes, la surexposition initiale étant significative pour 11 des 15 RPS considérés (tableau 2). Cette surexposition initiale n'est pas propre à une modalité particulière de mobilité, même si les RPS auxquels les travailleurs sont initialement surexposés varient suivant le type de mobilité. Les individus caractérisés par une mobilité externe volontaire apparaissent par exemple avant tout exposés au manque de liberté pour décider de la façon de faire leur travail ou aux mauvaises relations avec les collègues. De manière attendue, ceux caractérisés par une mobilité externe subie sont, en 2006, avant tout caractérisés par la peur de perdre leur emploi. Enfin, ceux qui connaitront dans les quatre années à venir une mobilité interne sont particulièrement exposés à la nécessité de penser à trop de choses à la fois ou à l'incapacité à employer pleinement leurs compétences.

Les prédictions montrent que cette surexposition ex-ante des individus connaissant une mobilité professionnelle dans leur emploi s'atténue considérablement dans leur nouvel emploi (tableau 2). Pour certains RPS, les individus connaissant une mobilité externe (voulue ou subie) sont même significativement moins exposés que ceux ne connaissant pas de mobilité. Ceux caractérisés par une mobilité interne demeurent en revanche surexposés pour une majorité de RPS.

Le tableau 3 présente l'effet net moyen de la mobilité professionnelle sur l'évolution de l'exposition, après contrôle des caractéristiques individuelles. Les effets sont calculés par la méthode de la double différence. Lorsque l'on considère indistinctement les types de mobilités, la mobilité professionnelle apparaît comme un facteur de réduction de l'exposition aux risques. Pour 6 RPS, la mobilité est associée à une diminution significative de l'exposition. De manière attendue, l'effet est particulièrement significatif lorsque la mobilité est voulue. Elle l'est aussi, mais dans une moindre mesure, lorsque la mobilité externe est subie, confirmant ainsi notre hypothèse et les résultats de l'enquête TNS du Conseil d'orientation pour l'emploi (2009). La mobilité subie a en particulier pour effet de réduire la peur de perdre son emploi. Ceux qui ont effectué une mobilité subie étaient en effet surexposés en 2006 au risque de perdre leur emploi (probabilité d'être exposé de $41 \%$ contre $28 \%$ pour ceux qui n'ont pas connu de mobilité). Le fait d'être à nouveau en emploi semble avoir rassuré une partie d'entre eux dans la mesure où en 2010 leur probabilité d'exposition s'est réduite de 11 points de pourcentage. Seul le sentiment de peur durant le travail augmente significativement suite à une mobilité externe subie. Lorsque la mobilité est interne à l'entreprise, l'effet est encore moins important mais n'est dans tous les cas jamais associé en moyenne à une augmentation de l'exposition, et cela même si une partie des mobilités internes peut être imposée par l'entreprise.

Le lien entre mobilités externes et RPS permet aussi de mieux comprendre l'exposition réduite des salariés en contrats à durée limitée par rapport aux salariés en CDI. Face à une exposition aux RPS, les salariés en CDI hésitent sans doute plus à effectuer une mobilité externe, dans la mesure où ils ont davantage à perdre que les salariés en contrats à durée limitée. Ils ont acquis des compétences spécifiques à l'entreprise qu'ils auront sans doute plus de mal à valoriser dans une autre entreprise. Ils ont davantage de perspectives de carrière dans l'entreprise. Ainsi il est probable qu'ils restent plus longtemps exposés aux RPS avant de parvenir à prendre la décision de quitter l'entreprise.

$$
* *
$$

La période 2006-2010 est marquée par une montée de l'exposition des travailleurs aux exigences du travail (après une pause dans l'intensification du travail, Coutrot et al., 2007), aux exigences émotionnelles, au manque d'autonomie et de marges de manœuvre, aux difficultés dans les rapports sociaux et relations de travail et aux conflits de valeur. Cette augmentation concerne ainsi l'ensemble des dimensions des RPS mises en évidence par le Collège d'expertise des risques psychosociaux (2011), à l'exception de l'insécurité socio-économique ressentie pour laquelle un effet de sélection des travailleurs les moins exposés semble 
Tableau 2

\section{Exposition aux RPS en 2006 et en 2010 suivant le type de mobilité professionnelle entre 2006} et 2010

A-Exposition en 2006

En \%

\begin{tabular}{|c|c|c|c|c|c|}
\hline (RPS 1) Travailler sous pression & 55 & $64^{\star \star \star}$ & $61^{\star \star}$ & $65^{\star \star \star}$ & $65^{\star \star \star}$ \\
\hline (RPS 2) Penser à trop de choses à la fois & 68 & $73^{\star *}$ & 66 & 71 & $76^{\star \star \star}$ \\
\hline (RPS 3) Effectuer une quantité de travail excessive & 51 & $59^{\star \star *}$ & $59^{* \star}$ & 53 & $62^{* * *}$ \\
\hline (RPS 4) Penser à son travail avant de dormir & 60 & 62 & 61 & 61 & 62 \\
\hline (RPS 5) Avoir du mal à concilier son travail avec ses obligations familiales & 27 & $32^{\star *}$ & 30 & 32 & $33^{\star \star \star}$ \\
\hline (RPS 6) Devoir cachez ses émotions, faire semblant d'être de bonne humeur & 56 & $59^{*}$ & 61 & 56 & 59 \\
\hline (RPS 7) Avoir peur pendant son travail & 30 & 31 & 33 & $23^{\star \star \star}$ & $34^{*}$ \\
\hline (RPS 8) Vivre des tensions avec un public & 36 & $41^{\star \star \star}$ & 40 & 39 & $40^{\star \star}$ \\
\hline (RPS 9) Ne pas pouvoir employer pleinement ses compétences & 34 & $45^{\star \star \star}$ & $45^{\star \star \star}$ & $42^{\star \star}$ & $45^{\star \star \star}$ \\
\hline (RPS10) Avoir très peu de liberté pour décider de la façon de faire son travail & 39 & 42 & $45^{\star \star}$ & 41 & 41 \\
\hline (RPS 11) Ne pas avoir un travail reconnu à sa juste valeur & 59 & $66^{\star \star \star}$ & $65^{\star \star}$ & 63 & $68^{\star \star \star}$ \\
\hline (RPS 12) Avoir de mauvaises relations avec ses collègues & 21 & $27^{\star \star \star}$ & $31^{\star \star \star}$ & $29^{\star \star}$ & $25^{\star \star}$ \\
\hline (RPS 13) Devoir faire des choses que l'on désapprouve & 30 & $37^{\star \star \star}$ & $40^{\star \star \star}$ & 32 & $37^{\star \star \star}$ \\
\hline (RPS 14) Ne pas avoir les moyens de faire un travail de qualité & 43 & $50^{\star \star \star}$ & $50^{\star \star}$ & 46 & $51^{\star \star \star}$ \\
\hline (RPS 15) Travailler avec la peur de perdre son emploi & 28 & 29 & 26 & $41^{\star \star \star}$ & 27 \\
\hline
\end{tabular}

\begin{tabular}{|c|c|c|c|c|c|}
\hline & \multicolumn{5}{|c|}{ Probabilité d'exposition en 2010} \\
\hline & \multirow{2}{*}{$\begin{array}{c}\text { Sans } \\
\text { mobilité } \\
\text { (ref) }\end{array}$} & \multicolumn{4}{|c|}{ Avec mobilité entre 2006 et 2010} \\
\hline & & Ensemble & $\begin{array}{c}\text { Externe } \\
\text { volontaire }\end{array}$ & $\begin{array}{l}\text { Externe } \\
\text { subie }\end{array}$ & Interne \\
\hline (RPS 1) Travailler sous pression & 65 & 65 & $58^{\star \star}$ & 62 & $69^{\star \star}$ \\
\hline (RPS 2) Penser à trop de choses à la fois & 74 & 76 & 70 & 75 & $79^{\star \star \star}$ \\
\hline (RPS 3) Effectuer une quantité de travail excessive & 57 & 58 & $51^{*}$ & 51 & $64^{\star \star \star}$ \\
\hline (RPS 4) Penser à son travail avant de dormir & 63 & 64 & $56^{*}$ & 63 & $67^{\star \star}$ \\
\hline (RPS 5) Avoir du mal à concilier son travail avec ses obligations familiales & 36 & $39^{*}$ & 40 & 41 & $39^{*}$ \\
\hline (RPS 6) Devoir cachez ses émotions, faire semblant d'être de bonne humeur & 62 & 63 & 58 & 61 & 64 \\
\hline (RPS 7) Avoir peur pendant son travail & 30 & 30 & 27 & 31 & 32 \\
\hline (RPS 8) Vivre des tensions avec un public & 39 & 40 & 40 & $32^{\star *}$ & 41 \\
\hline (RPS 9) Ne pas pouvoir employer pleinement ses compétences & 45 & 48 & 40 & 46 & $51^{\star \star \star}$ \\
\hline (RPS10) Avoir très peu de liberté pour décider de la façon de faire son travail & 41 & 41 & $30^{\star \star \star}$ & 42 & $47^{\star \star \star}$ \\
\hline (RPS 11) Ne pas avoir un travail reconnu à sa juste valeur & 76 & 77 & $69^{\star \star}$ & 76 & $81^{\star \star \star}$ \\
\hline (RPS 12) Avoir de mauvaises relations avec ses collègues & 32 & 35 & 32 & 28 & $38^{\star \star \star}$ \\
\hline (RPS 13) Devoir faire des choses que l'on désapprouve & 36 & 37 & 34 & 32 & 39 \\
\hline (RPS 14) Ne pas avoir les moyens de faire un travail de qualité & 51 & $55^{\star \star}$ & 49 & 51 & $59^{\star \star \star}$ \\
\hline (RPS 15) Travailler avec la peur de perdre son emploi & 26 & 24 & 22 & 30 & 26 \\
\hline
\end{tabular}

Note : ces probabilités ont été prédites à partir des résultats d'estimation de la spécification 2 incluant des indicatrices de mobilité et estimées sur l'échantillon restreint des individus enquêtés en 2006 et $2010 .{ }^{*},{ }^{\star \star}{ }^{*}$ et ${ }^{* \star \star}$ indiquent que la probabilité est significativement différente de la probabilité sans mobilité respectivement aux seuils de $1 \%, 5 \%$ et $10 \%$.

Lecture : en 2006, un individu n'ayant pas connu de mobilité professionnelle entre 2006 et 2010 a en moyenne une probabilité égale à $55 \%$ d'être exposé au RPS 1 (Travailler sous pression). Toutes choses égales par ailleurs, cette même probabilité est égale à $61 \%$ en cas de mobilité externe voulue, $65 \%$ en cas de mobilité externe subie et $65 \%$ en case de mobilité interne. Quel que soit le type de mobilité, la différence d'exposition est significativement différente de l'exposition sans mobilité professionnelle.

Champ : individus âgés de 20 à 68 ans, déclarant exercer une activité professionnelle en tant que salarié d'une entreprise, d'un artisan, d'une association, d'un particulier, ou en tant que chef d'entreprise ou indépendant, enquêtés en 2006 et 2010.

Source : SIP 2006 et 2010. 
l'emporter sur l'effet potentiel de la crise économique sur l'insécurité de l'emploi ressentie par les travailleurs. L'analyse des déterminants de l'exposition et de son évolution met en lumière au niveau individuel un effet de persistance dans le temps et une diversité des déterminants selon le type de RPS. Les populations les plus exposées se distinguent au moins partiellement des groupes souvent considérés comme les plus fragiles sur le marché du travail (jeunes, seniors, non diplômés, salariés en CDD ou intérim). Les moins de 35 ans, les plus de 45 ans et ceux ayant un diplôme inférieur au baccalauréat sont moins exposés à la quasi-totalité des RPS. Avoir un emploi à durée limitée réduit l'exposition aux exigences du travail, aux mauvaises relations de travail ou aux conflits de valeur. De manière plus attendue, les individus ayant un travail physiquement exigeant, répétitif ou à la chaîne et ceux ayant un travail posté ou de nuit sont globalement plus exposés. Ces résultats soulignent l'intérêt d'adapter les politiques de prévention des RPS afin de cibler les populations les plus exposées.
Les différents indicateurs d'exposition à la crise construits au niveau des secteurs d'activité n'apparaissent pas significatifs pour rendre compte de l'évolution de l'exposition des travailleurs aux RPS. Il se peut donc que la perception de la crise et de ses effets soit relativement hétérogène au sein d'un même secteur d'activité, soit parce que toutes les entreprises ne sont pas touchées de la même manière par l'évolution de l'activité, soit parce que face à une même réduction de l'activité les réactions des travailleurs diffèrent. Le lien positif entre le fait d'appartenir à une entreprise qui a mis en place un plan de licenciement et l'exposition aux RPS semble confirmer la première partie de cette hypothèse. Le lien entre mobilité professionnelle et exposition aux RPS donne de la consistance à la seconde partie de cette hypothèse. Dans la mesure où la crise s'est accompagnée d'une baisse des mobilités externes, et étant donné le lien mis en évidence entre mobilité d'un emploi vers un autre et réduction de l'exposition aux RPS, l'aggravation de l'exposition entre 2006 et 2010 peut en partie s'expliquer par une moindre

Tableau 3

Effet net de la mobilité sur l'évolution de l'exposition aux RPS entre 2006 et 2010

\begin{tabular}{|c|c|c|c|c|}
\hline & \multicolumn{4}{|c|}{ en point de pourcentage } \\
\hline & \multirow[b]{2}{*}{$\begin{array}{c}\text { Tout type } \\
\text { de mobilité }\end{array}$} & \multicolumn{3}{|c|}{ Par type de mobilité } \\
\hline & & $\begin{array}{c}\text { Externe } \\
\text { volontaire }\end{array}$ & $\begin{array}{l}\text { Externe } \\
\text { subie }\end{array}$ & Interne \\
\hline (RPS 1) Travailler sous pression & $-10^{\star \star *}$ & $-13^{\star \star *}$ & $-13^{\star \star *}$ & $-6^{\star *}$ \\
\hline (RPS 2) Penser à trop de choses à la fois & ns & ns & ns & ns \\
\hline (RPS 3) Effectuer une quantité de travail excessive & $-6^{\star \star \star}$ & $-13^{\star \star \star}$ & ns & ns \\
\hline (RPS 4) Penser à son travail avant de dormir & ns & $-7^{\star *}$ & ns & ns \\
\hline (RPS 5) Avoir du mal à concilier son travail avec ses obligations familiales & ns & ns & ns & ns \\
\hline (RPS 6) Devoir cachez ses émotions, faire semblant d'être de bonne humeur & ns & $-8^{\star *}$ & ns & ns \\
\hline (RPS 7) Avoir peur pendant son travail & ns & $-6^{*}$ & $+8^{\star \star}$ & ns \\
\hline (RPS 8) Vivre des tensions avec un public & $-4^{\star}$ & ns & $-11^{\star \star \star}$ & ns \\
\hline (RPS 9) Ne pas pouvoir employer pleinement ses compétences & $-9^{\star \star \star}$ & $-16^{\star \star \star}$ & ns & $-5^{\star}$ \\
\hline (RPS10) Avoir très peu de liberté pour décider de la façon de faire son travail & ns & $-17^{\star \star \star}$ & ns & ns \\
\hline (RPS 11) Ne pas avoir un travail reconnu à sa juste valeur & $-6^{\star \star \star}$ & $-13^{\star \star \star}$ & ns & ns \\
\hline (RPS 12) Avoir de mauvaises relations avec ses collègues & ns & $-10^{\star \star \star}$ & $-10^{\star \star \star}$ & ns \\
\hline (RPS 13) Devoir faire des choses que l'on désapprouve & $-6^{\star \star \star}$ & $-12^{\star \star \star}$ & ns & ns \\
\hline (RPS 14) Ne pas avoir les moyens de faire un travail de qualité & ns & $-10^{\star \star \star}$ & ns & ns \\
\hline (RPS 15) Travailler avec la peur de perdre son emploi & ns & ns & $-8^{\star \star \star}$ & ns \\
\hline
\end{tabular}

Note : les effets moyens présentés ont été estimés à partir des résultats d'estimation de la spécification 2 du modèle incluant des indicatrices de mobilités professionnelles et estimé sur l'échantillon restreint des individus en emploi enquêtés en 2006 et 2010 . ${ }^{*},{ }^{* *}$ et ${ }^{* * *}$ indique que l'évolution moyenne est significativement différente de l'évolution de référence (sans mobilité) respectivement aux seuils de $1 \%, 5 \%$ et $10 \%$.

Lecture : toutes choses égales par ailleurs, un individu ayant connu une mobilité professionnelle entre 2006 et 2010 connaît en moyenne une évolution de l'exposition au RPS 1 (Travailler sous pression) inférieure de 10 points de pourcentage à l'évolution caractérisant un individu n'ayant pas connu de mobilité. La différence est significative au seuil de $1 \%$.

Champ : individus âgés de 20 à 68, déclarant exercer une activité professionnelle en tant que salarié d'une entreprise, d'un artisan, d'une association, d'un particulier, ou en tant que chef d'entreprise ou indépendant, enquêtés en 2006 et 2010.

Source : SIP 2006 et 2010. 
capacité des travailleurs à échapper, par une mobilité professionnelle externe, à des situations d'exposition à des RPS au travail.

La mise en évidence sur la période 2006-2010 d'une exposition plus fréquente des travailleurs aux RPS s'accompagne d'une dégradation de leur état de santé (Gaillard et al., 2016). Les effets des RPS sur la santé peuvent entraîner des incapacités durables pour une partie des individus, qui sont de nature à compromettre leur capacité à reculer l'âge de leur passage à la retraite, à dégrader leurs conditions de vie et à accroître les dépenses de santé, et donc d'importants coûts sociaux (cf. Béjean et Sultan-Taïeb, 2005, Sultan-Taïeb et al., 2013 pour des mesures des coûts du stress professionnel). Lorsqu'on se cantonne au périmètre de l'emploi, ce phénomène peut apparaître compensé par un effet de sélection des travailleurs en meilleure santé lié à la crise. Néanmoins la prise en compte des conséquences au-delà du périmètre des travailleurs qui restent en emploi plaide pour un renforcement des politiques de prévention des RPS et des politiques de formation susceptibles de favoriser une plus grande mobilité des travailleurs afin de limiter les expositions aux RPS.

\section{BIBLIOGRAPHIE}

Amar A., Dalibard E. et Debauche E. (2011), « La crise de 2008-2009 et ses suites : recul marqué de l'emploi et ralentissement des salaires puis reprise en 2010 », dans Insee Emploi et Salaires, Insee Référence - édition 2011, pp. 9-17.

Amossé T. (2003), «Interne ou externe : deux visages de la mobilité professionnelle», Insee Première, $\mathrm{n}^{\circ}$ 921, septembre.

Amossé T. et Gollac M. (2008), «Intensité du travail et mobilité professionnelle », Travail et Emploi, $\mathrm{n}^{\circ} 113$, pp. 59-73.

Ananian S., Debauche E. et Prost C. (2012), «L'ajustement du marché du travail français pendant la crise de 2008-2009», Dares Analyses, n 040.

Argouarc'h J., Debauche E., Leblanc P. et Ourliac B. (2010), «Comment expliquer les évolutions de l'emploi depuis le début de la crise ?», Dossier de la Note de conjoncture, Insee.

Asfaw A., Pana-Cryan R. et Rosa R. (2011), " The business cycle and the incidence of workplace injuries: evidence from the U.S.A », Journal of Safety Research, vol. 42, n 1, pp. 1-8.

Béjean S. et Sultan-Taïeb H. (2005), « Modeling the economic burden of diseases imputable to stress at work », European Journal of Health Economics, vol. 6, $\mathrm{n}^{\circ}$ 1, pp. 16-123.

Bender K. A., Economou A. et Theodossiou I. (2013), «The temporary and permanent effects of unemployment on mortality in Europe », International Labour Review, vol. 152, n 2, pp. 275-286.
Beque M. (2014), «Les risques psychosociaux au travail. Un panorama d'après l'enquête Santé et itinéraire professionnel 2010 », Dares Analyses $n^{\circ} 31$.

Bonde J. P. (2008), « Psychosocial factors at work and risk of depression: a systematic review of the epidemiological evidence », Occupational Environmental Medicine, vol. 5, n 7, pp. 38-445.

Boone J. et van Ours J. C. (2006), « Are Recessions Good for Workplace Safety? », Journal of Health Economics, vol. 25, n 6, pp. 1069-1093.

Boone J., van Ours J. C., Wuellrich J. P. et Zweimuller J. (2011), " Recessions Are Bad for Workplace Safety », Journal of Health Economics, vol. 30, $\mathrm{n}^{\circ} 4$, pp. 764-773.

Brenner M. H. (1987), « Relation to Economic Change to Swedish Health and Social Well-being, 1950-1980», Social Science and Medicine, vol. $25, \mathrm{n}^{\circ} 2$, pp. 183-195.

Buchmueller J., Grignon M. et Jusot F. (2007), «Unemployment and Mortality in France 1982-2002 », CHEPA Working Paper Serie, $\mathrm{n}^{\circ}$ 07-04, MacMaster University.

Bué J., Coutrot T., Guignon N. et Sandret N. (2008), «Les facteurs de risques psychosociaux au travail. Une approche quantitative par l'enquête Sumer ", Revue française des affaires sociales, $\mathrm{n}^{\circ} 2-3$, pp. 45-70.

Catalano R. et Dooley D. (1983), « Health effects of economic instability: A test of the economic 
stress hypothesis », Jounal of Health and Social Behaviour, vol. 24, n 1, pp. 46-60.

Centre d'analyse stratégique (2009), « L'ajustement de l'emploi dans la crise : la flexibilité sans la mobilité ? », La note de veille, $\mathrm{n}^{\circ} 156$.

Chamberlain G (1980), «Analysis of covariance with qualitative data ", Review of Economic Studies, $\mathrm{n}^{\circ} 47$, pp. 225-238.

Charles K. K. et DeCicca P. (2008), « Local Labor Market Fluctuations and Health: Is There a Connection and for Whom? », Journal of Health Economics, vol. 27, $\mathrm{n}^{\circ}$ 6, pp. 1532-1550.

Cochard M., Cornilleau G. et Heyer E. (2010), « Les marchés du travail dans la crise », Économie et statistique, $\mathrm{n}^{\circ}$ 438-440, pp. 181-204.

Coile C., Levine P. B. et McKnight R. (2012), « Recessions, Older Workers, and Longevity: How Long Are Recessions Good For Your Health? », NBER Working Paper, $\mathrm{n}^{\circ} 18361$.

Collège d'expertise sur le suivi des risques psychosociaux au travail (2011), "Mesurer les facteurs psychosociaux de risque au travail pour les maîtriser", téléchargeable sur http://www.ladocumentationfrancaise.fr/rapports-publics/114000201/index.shtml.

Conseil d'orientation pour l'emploi (2009), Rapport sur les trajectoires et les mobilités professionnelles.

Coutrot T., Bué J., Hamon-Cholet S. et Vinck L. (2007), «Conditions de travail : une pause dans l'intensification du travail », Premières Synthèses, $\mathrm{n}^{\circ} 01.2$, Dares.

Coutrot T. et Mermilliod C. (2010), «Les risques psychosociaux au travail : les indicateurs disponibles », Dares Analyses, $\mathrm{n}^{\circ} 80$.

Dares (2015), «Le taux de rotation de la main-d'œuvre poursuit sa hausse au $1^{\text {er }}$ trimestre $2015 »$, Dares Indicateur, $\mathrm{n}^{\circ} 54$.

Davies R., Jones P. et Nunez I. (2009), « The impact of the business cycle on occupational injuries in the UK », Social Science \& Medicine, vol. $69, \mathrm{n}^{\circ} 2$, pp. 178-182.

Fenwick R. et Tausig M. (1994), « The macroeconomic context of job stress », Journal of Health and Social Behavior, vol. 35, $\mathrm{n}^{\circ} 3$, pp. 266-282.
Ferrie J. E., Shipley M. J., Marmot M., Standfeld S. A. et Smith G. D. (1998), « The health effects of major organisational change and job insecurity », Social Science and Medicine, vol. 46, n 2, pp. 243-254.

Ferrie J. E., Shipley M. J., Newman K., Stanfeld S. A. et Marmot M. (2005), "SelfReported Job Insecurity and Health in the Whitehall II Study: Potential Explanations of the Relationship », Social Science and Medicine, vol. $60, \mathrm{n}^{\circ} 7$, pp. 1593-1602.

Ferrie J. E., Westerlund H., Oxenstierna G. et Theorell T. (2007), « The impact of moderate and major workplace expansion and downsizing on the psychosocial and physical work environment and income in Sweden ». Scandinavian Journal of Public Health, vol. 35, $\mathrm{n}^{\circ}$ 1, pp. 62-69.

Gaillard A., Fontaine R. et Sauze D. (2016), «Psychosocial risks exposure and mental health status: analysis from SIP survey », Document de travail LEDI, à paraître.

Greene W. H. (2004), Econometric Analysis (Fourth Edition), Prentice-Hall, Pearson Education, New Jersey,

Karasek R. A. et Theorell T. (1990), Healthy work: Stress, productivity, and the reconstruction of working life, Basic Book, New-York.

Kieselbach T. et Triomphe C. E. (éds.) (2010), Santé et Restructurations (HIRES): Recommandations, réponses nationales et questions politiques dans l'UE, Rainer Hampp Verlag.

Kivimaki M., Vahtera J., Ferrie J. E., Hemingway H., Pentti J. (2001), «Organisational downsizing and musculoskeletal problems in employees: a prospective study ». Occupational \& Environmental Medicine, vol. 58, $\mathrm{n}^{\circ} 12$, pp. 811-817.

Lalé E. (2010), «Une mesure de la mobilité professionnelle en France de 1982 à 2007 », Les mobilités des salariés, Rapport du CAE, $\mathrm{n}^{\circ} 91$, pp. 205-217.

Lollivier S. (2006), Économétrie avancée des variables qualitatives, Economica, Collection Economie et Statistiques Avancées.

Marchand O. (2010), « Panorama statistique de la mobilité professionnelle en France », dans Les mobilités des salariés, Rapport du CAE, $\mathrm{n}^{\circ} 91$, pp. 181-196. 
McInerney M., Mellor J.M. (2012), « Recessions and Seniors' Health, Health Behaviors, and Healthcare Use: Analysis of the Medicare Current Beneficiary Survey ». Journal of Health Economics, vol. 31, n 5, pp. 744-751.

Netterstrom B., Conrad N., Bech P. et al. (2008), «The Relation between Work-related Psychosocial Factors and the Development of Depression », Epidemiologic Reviews, vol. 30, $\mathrm{n}^{\circ} 1$, pp. 118-132.

Niedhammer I., Chastang J.-F., Levy D., David S. et Degioanni S. (2007), « Exposition aux facteurs psychosociaux au travail du modèle de Karasek en France : étude méthodologique à l'aide de l'enquête nationale SUMER », Travailler, $\mathrm{n}^{\circ} 17$, p. 47-70.

Noer D. M. (1997), « Layoff survivor sickness : What it is and what to do about it», dans M. K. Gowing, J. D. Kraft et J. C. Quick (éds), The new organizational reality: Downsizing, restructuring and revitalization, Washington (DC), American Psychological Association.

Perez C. et Thevenot N. (coord.) (2014), « Modes d'ajustement à la crise : des relations professionnelles sous tensions ». Documents de travail $d u$ Centre d'économie de la Sorbonne, n²014-76.

Poirier D. J. et Rudd P. A. (1988), « Probit with Dependent Observations », The Review of Economic Studies, vol. 55, n 4, pp. 593-614.

Robinson J. C., Shor G. M. (1989), « Business-cycle influences on work-related disability in construction and manufacturing ", The Mil- bank Quartely, vol. 67, supplément 2, partie 2, pp. 92-113.

Ruhm C. J. (2003), « Good Times Make You Sick », Journal of Health Economics, vol. 22, $\mathrm{n}^{\circ} 4$, pp. 637-658.

Ruhm C. J. (2004), « Macroeconomic Conditions, Health and Mortality », NBER working paper, $\mathrm{n}^{\circ} 11007$.

Siegrist J. (1996), «Adverse health effects of high-effort/low-reward conditions », Journal of Occupational Health Psychology, vol. 1, $\mathrm{n}^{\circ} 1$, pp. 27-41.

Söderberg M., Härenstam A., Rosengren A., Schiöler L., Olin A. C., Lissner L. et Torén K. (2014), «Psychosocial work environment, job mobility and gender differences in turnover behaviour: a prospective study among the Swedish general population », BMC public health, vol. 14, pp. 605 .

Sultan-Taïeb H., Chastang J.-F., Mansouri M., Niedhammer I., (2013), "The annual costs of cardiovascular diseases and mental disorders attributable to job strain in France », BMC Public Health, vol. 13, p. 748.

Ussif A. A. (2004), « An International Analysis of Workplace Injuries », Monthly Labor Review, vol. $127, \mathrm{n}^{\circ} 3$, pp. 41-51.

Yahou N. et Bouvet M. (2001), « Le risque d'accidents du travail varie avec la conjoncture économique », Premières Informations-Premières Synthèses, $\mathrm{n}^{\circ} 31-1$, Dares. 


\section{RÉSULTATS D'ESTIMATIONS : EFFET DES CARACTÉRISTIQUES INDIVIDUELLES SUR L'EXPOSITION AU RPS ET SUR L'ÉVOLUTION DE L'EXPOSITION AUX RPS ENTRE 2006 ET 2010}

Tableau A

Effets marginaux des caractéristiques individuelles sur la probabilité d'être exposé aux RPS

\begin{tabular}{|c|c|c|c|c|c|c|}
\hline & & \multicolumn{5}{|c|}{$\begin{array}{c}\text { DIM } 1 \\
\text { Exigences du travail }\end{array}$} \\
\hline & & RPS 1 & RPS 2 & RPS 3 & RPS 4 & RPS 5 \\
\hline \multicolumn{2}{|l|}{ Indicatrices du secteur d'activité } & Oui & Oui & Oui & Oui & Oui \\
\hline $\begin{array}{l}\text { Sexe } \\
\text { Réf: Homme }\end{array}$ & Femme & $\begin{array}{r}-0,01 \\
(0,20)\end{array}$ & $\begin{array}{c}0,02 \\
(0,12)\end{array}$ & $\begin{array}{l}0,03^{\star *} \\
(0,02)\end{array}$ & $\begin{array}{l}0,05^{\star \star \star} \\
(0,00)\end{array}$ & $\begin{array}{l}0,04^{\star \star *} \\
(0,00)\end{array}$ \\
\hline \multirow[t]{3}{*}{$\begin{array}{l}\text { Âge } \\
\text { Réf : }[35 ; 44]\end{array}$} & {$[-; 34]$} & $\begin{array}{c}-0,03^{\star \star} \\
(0,02)\end{array}$ & $\begin{array}{l}-0,01 \\
(0,50)\end{array}$ & $\begin{array}{r}-0,01 \\
(0,51)\end{array}$ & $\begin{array}{c}0,00 \\
(0,96)\end{array}$ & $\begin{array}{l}-0,06^{\star \star *} \\
(0,00)\end{array}$ \\
\hline & {$[45 ; 54]$} & $\begin{array}{r}-0,02^{*} \\
(0,08)\end{array}$ & $\begin{array}{c}-0,03^{\star \star \star} \\
(0,00)\end{array}$ & $\begin{array}{c}-0,02^{\star *} \\
(0,05)\end{array}$ & $\begin{array}{c}0,00 \\
(0,87)\end{array}$ & $\begin{array}{l}-0,07^{\star * *} \\
(0,00)\end{array}$ \\
\hline & {$[55 ;+]$} & $\begin{array}{c}-0,06^{\star \star *} \\
(0,00)\end{array}$ & $\begin{array}{l}-0,06^{\star \star \star} \\
(0,00)\end{array}$ & $\begin{array}{c}-0,06^{\star \star *} \\
(0,00)\end{array}$ & $\begin{array}{r}-0,01 \\
(0,56)\end{array}$ & $\begin{array}{l}-0,14^{\star * *} \\
(0,00)\end{array}$ \\
\hline \multirow[t]{4}{*}{$\begin{array}{l}\text { Education } \\
\text { Réf : Baccalauréat }\end{array}$} & $\begin{array}{l}\text { Aucun diplôme, CEP, brevet, } \\
\text { BEPC }\end{array}$ & $\begin{array}{c}-0,20^{\star \star \star} \\
(0,00)\end{array}$ & $\begin{array}{c}-0,09^{\star \star \star} \\
(0,00)\end{array}$ & $\begin{array}{c}-0,07^{\star \star \star} \\
(0,00)\end{array}$ & $\begin{array}{c}-0,09^{\star \star \star} \\
(0,00)\end{array}$ & $\begin{array}{c}-0,14^{\star \star \star} \\
(0,00)\end{array}$ \\
\hline & CAP, BEP & $\begin{array}{c}-0,17^{\star \star \star} \\
(0,00)\end{array}$ & $\begin{array}{l}-0,06^{\star \star \star} \\
(0,00)\end{array}$ & $\begin{array}{c}-0,04^{\star \star \star} \\
(0,00)\end{array}$ & $\begin{array}{c}-0,06^{\star \star \star} \\
(0,00)\end{array}$ & $\begin{array}{c}-0,12^{\star \star \star} \\
(0,00)\end{array}$ \\
\hline & Bac+2 ou sup. & $\begin{array}{c}-0,08^{\star \star \star} \\
(0,00)\end{array}$ & $\begin{array}{r}-0,02 \\
(0,25)\end{array}$ & $\begin{array}{r}-0,01 \\
(0,52)\end{array}$ & $\begin{array}{r}-0,01 \\
(0,71)\end{array}$ & $\begin{array}{c}-0,06^{\star \star \star} \\
(0,00)\end{array}$ \\
\hline & Non renseigné & $\begin{array}{c}-0,10^{\star * \star} \\
(0,00)\end{array}$ & $\begin{array}{c}-0,04^{\star \star \star} \\
(0,01)\end{array}$ & $\begin{array}{r}-0,02 \\
(0,28)\end{array}$ & $\begin{array}{c}-0,04^{\star *} \\
(0,02)\end{array}$ & $\begin{array}{l}-0,08^{\star \star \star} \\
(0,00)\end{array}$ \\
\hline \multirow[t]{6}{*}{$\begin{array}{l}\text { PCS } \\
\text { Réf : Employé }\end{array}$} & Agriculteur & $\begin{array}{c}0,02 \\
(0,69)\end{array}$ & $\begin{array}{l}0,07^{*} \\
(0,07)\end{array}$ & $\begin{array}{r}-0,03 \\
(0,47)\end{array}$ & $\begin{array}{l}0,11^{\star \star \star} \\
(0,01)\end{array}$ & $\begin{array}{l}0,03 \\
(0,42)\end{array}$ \\
\hline & $\begin{array}{l}\text { Artisan, commerçant, chef } \\
\text { d'entreprise }\end{array}$ & $\begin{array}{c}0,03 \\
(0,25)\end{array}$ & $\begin{array}{l}0,07^{\star \star \star} \\
(0,01)\end{array}$ & $\begin{array}{r}-0,03 \\
(0,38)\end{array}$ & $\begin{array}{l}0,15^{\star \star \star} \\
(0,00)\end{array}$ & $\begin{array}{c}0,03 \\
(0,26)\end{array}$ \\
\hline & Cadre et profession intellectuelle & $\begin{array}{l}0,15^{\star \star \star} \\
(0,00)\end{array}$ & $\begin{array}{l}0,13^{\star \star \star} \\
(0,00)\end{array}$ & $\begin{array}{l}0,13^{\star \star \star} \\
(0,00)\end{array}$ & $\begin{array}{l}0,17^{\star \star \star} \\
(0,00)\end{array}$ & $\begin{array}{l}0,16^{\star \star *} \\
(0,00)\end{array}$ \\
\hline & Profession intermédiaires & $\begin{array}{l}0,09^{\star \star *} \\
(0,00)\end{array}$ & $\begin{array}{l}0,11^{\star \star \star} \\
(0,00)\end{array}$ & $\begin{array}{l}0,00^{\star \star *} \\
(0,00)\end{array}$ & $\begin{array}{l}0,10^{\star \star \star} \\
(0,00)\end{array}$ & $\begin{array}{l}0,04^{* \star *} \\
(0,00)\end{array}$ \\
\hline & Ouvrier & $\begin{array}{r}-0,11 \\
(0,00)\end{array}$ & $\begin{array}{l}-0,12^{\star \star \star} \\
(0,00)\end{array}$ & $\begin{array}{c}-0,07^{\star \star *} \\
(0,00)\end{array}$ & $\begin{array}{l}-0,07^{\star \star \star} \\
(0,00)\end{array}$ & $\begin{array}{c}-0,06^{\star \star \star} \\
(0,00)\end{array}$ \\
\hline & Non renseigné & $\begin{array}{c}-0,15^{\star \star \star} \\
(0,00)\end{array}$ & $\begin{array}{l}-0,23^{\star \star \star} \\
(0,00)\end{array}$ & $\begin{array}{c}-0,09^{\star \star *} \\
(0,00)\end{array}$ & $\begin{array}{l}-0,12^{\star \star \star} \\
(0,00)\end{array}$ & $\begin{array}{c}-0,07^{\star * \star} \\
(0,00)\end{array}$ \\
\hline \multirow[t]{3}{*}{$\begin{array}{l}\text { Contrat de travail } \\
\text { Réf : Sans limitation de durée }\end{array}$} & $\begin{array}{l}\text { Durée limitée (contrat à durée } \\
\text { déterminée ou intérim) }\end{array}$ & $\begin{array}{c}-0,07^{\star \star \star} \\
(0,00)\end{array}$ & $\begin{array}{c}-0,08^{\star \star \star} \\
(0,00)\end{array}$ & $\begin{array}{l}-0,10^{\star \star \star} \\
(0,00)\end{array}$ & $\begin{array}{r}-0,01 \\
(0,57)\end{array}$ & $\begin{array}{r}-0,02 \\
(0,12)\end{array}$ \\
\hline & Autres (saisonniers...) & $\begin{array}{r}-0,03 \\
(0,28)\end{array}$ & $\begin{array}{r}-0,04^{*} \\
(0,07)\end{array}$ & $\begin{array}{r}-0,04 \\
(0,11)\end{array}$ & $\begin{array}{r}-0,03 \\
(0,23)\end{array}$ & $\begin{array}{r}-0,03 \\
(0,11)\end{array}$ \\
\hline & Indépendant & $\begin{array}{c}-0,09^{\star \star *} \\
(0,00)\end{array}$ & $\begin{array}{r}-0,04 \\
(0,11)\end{array}$ & $\begin{array}{c}-0,20^{\star \star \star} \\
(0,00)\end{array}$ & $\begin{array}{r}-0,03 \\
(0,25)\end{array}$ & $\begin{array}{c}0,01 \\
(0,69)\end{array}$ \\
\hline $\begin{array}{l}\text { Temps partiel } \\
\text { Réf : Non }\end{array}$ & Oui & $\begin{array}{c}-0,05^{\star \star \star} \\
(0,00)\end{array}$ & $\begin{array}{l}-0,06^{\star \star \star} \\
(0,00)\end{array}$ & $\begin{array}{c}-0,07^{\star \star \star} \\
(0,00)\end{array}$ & $\begin{array}{c}-0,03^{\star \star \star} \\
(0,01)\end{array}$ & $\begin{array}{r}-0,01 \\
(0,65)\end{array}$ \\
\hline $\begin{array}{l}\text { Temps de travail souvent } \\
\text { supérieur à } 48 \mathrm{~h} \text { par semaine } \\
\text { Réf : Non }\end{array}$ & Oui & $\begin{array}{l}0,11^{* \star *} \\
(0,00)\end{array}$ & $\begin{array}{l}0,13^{\star \star \star} \\
(0,00)\end{array}$ & $\begin{array}{l}0,13^{\star \star \star} \\
(0,00)\end{array}$ & $\begin{array}{l}0,13^{\star \star \star} \\
(0,00)\end{array}$ & $\begin{array}{l}0,17^{\star \star *} \\
(0,00)\end{array}$ \\
\hline $\begin{array}{l}\text { Travail posté } \\
\text { Réf : Non }\end{array}$ & Oui & $\begin{array}{c}0,01 \\
(0,60)\end{array}$ & $\begin{array}{c}0,00 \\
(0,88)\end{array}$ & $\begin{array}{c}0,00 \\
(0,99)\end{array}$ & $\begin{array}{l}0,04^{\star \star \star} \\
(0,00)\end{array}$ & $\begin{array}{l}0,08^{\star \star *} \\
(0,00)\end{array}$ \\
\hline $\begin{array}{l}\text { Travail de nuit } \\
\text { Réf : Non }\end{array}$ & Oui & $\begin{array}{c}0,02 \\
(0,23)\end{array}$ & $\begin{array}{c}0,00 \\
(0,81)\end{array}$ & $\begin{array}{c}0,02 \\
(0,18)\end{array}$ & $\begin{array}{r}-0,02 \\
(0,19)\end{array}$ & $\begin{array}{c}0,01 \\
(0,47)\end{array}$ \\
\hline $\begin{array}{l}\text { Travail répétitif ou à la chaine } \\
\text { Réf : Non }\end{array}$ & Oui & $\begin{array}{l}0,13^{\star \star \star} \\
(0,00)\end{array}$ & $\begin{array}{l}0,04^{\star \star \star} \\
(0,00)\end{array}$ & $\begin{array}{l}0,13^{\star \star \star} \\
(0,00)\end{array}$ & $\begin{array}{l}0,02^{\star *} \\
(0,04)\end{array}$ & $\begin{array}{l}0,06^{\star \star *} \\
(0,00)\end{array}$ \\
\hline $\begin{array}{l}\text { Travail physiquement exigeant } \\
\text { Réf : Non }\end{array}$ & Oui & $\begin{array}{l}0,12^{\star \star \star} \\
(0,00)\end{array}$ & $\begin{array}{l}0,09^{\star \star \star} \\
(0,00)\end{array}$ & $\begin{array}{l}0,15^{\star \star \star} \\
(0,00)\end{array}$ & $\begin{array}{l}0,06^{\star \star \star} \\
(0,00)\end{array}$ & $\begin{array}{l}0,07^{\star \star *} \\
(0,00)\end{array}$ \\
\hline
\end{tabular}


Tableau A (suite)

\begin{tabular}{|c|c|c|c|c|c|c|}
\hline & \multicolumn{3}{|c|}{$\begin{array}{c}\text { DIM } 2 \\
\text { Exigences émotionnelles }\end{array}$} & \multicolumn{2}{|c|}{$\begin{array}{c}\text { DIM } 3 \\
\text { Manque d'autonomie } \\
\text { et marges } \\
\text { de manœuvre }\end{array}$} \\
\hline & & RPS 6 & RPS 7 & RPS 8 & RPS 9 & RPS 10 \\
\hline \multicolumn{2}{|l|}{ Indicatrices du secteur d'activité } & Oui & Oui & Oui & Oui & Oui \\
\hline $\begin{array}{l}\text { Sexe } \\
\text { Réf : Homme }\end{array}$ & femme & $\begin{array}{l}0,09^{\star \star \star} \\
(0,00)\end{array}$ & $\begin{array}{c}-0,05^{\star \star \star} \\
(0,00)\end{array}$ & $\begin{array}{c}-0,01 \\
(0,45)\end{array}$ & $\begin{array}{c}0,00 \\
(0,87)\end{array}$ & $\begin{array}{l}0,00 \\
(0,70)\end{array}$ \\
\hline \multirow[t]{3}{*}{$\begin{array}{l}\text { Âge } \\
\text { Réf : [35; 44] }\end{array}$} & {$[-; 34]$} & $\begin{array}{c}-0,01 \\
(0,37)\end{array}$ & $\begin{array}{c}0,01 \\
(0,24)\end{array}$ & $\begin{array}{c}0,01 \\
(0,35)\end{array}$ & $\begin{array}{c}0,02^{*} \\
(0,08)\end{array}$ & $\begin{array}{l}0,04^{\star \star \star} \\
(0,00)\end{array}$ \\
\hline & {$[45 ; 54]$} & $\begin{array}{c}0,02 \\
(0,16)\end{array}$ & $\begin{array}{c}0,00 \\
(0,86)\end{array}$ & $\begin{array}{c}-0,02^{\star *} \\
(0,05)\end{array}$ & $\begin{array}{c}-0,01 \\
(0,22)\end{array}$ & $\begin{array}{c}-0,01 \\
(0,41)\end{array}$ \\
\hline & {$[55 ;+]$} & $\begin{array}{l}0,01 \\
(0,49)\end{array}$ & $\begin{array}{r}-0,02^{*} \\
(0,07)\end{array}$ & $\begin{array}{l}-0,05^{\star \star \star} \\
(0,00)\end{array}$ & $\begin{array}{c}-0,05^{\star \star *} \\
(0,00)\end{array}$ & $\begin{array}{c}-0,03^{* *} \\
(0,02)\end{array}$ \\
\hline \multirow[t]{4}{*}{$\begin{array}{l}\text { Éducation } \\
\text { Réf : Baccalauréat }\end{array}$} & Aucun dipl., CEP, brevet, BEPC & $\begin{array}{c}-0,10^{\star \star \star} \\
(0,00)\end{array}$ & $\begin{array}{l}0,01 \\
(0,59)\end{array}$ & $\begin{array}{c}-0,10^{\star \star *} \\
(0,00)\end{array}$ & $\begin{array}{c}-0,12^{\star \star *} \\
(0,00)\end{array}$ & $\begin{array}{c}-0,02 \\
(0,21)\end{array}$ \\
\hline & CAP, BEP & $\begin{array}{c}-0,07^{\star \star \star} \\
(0,00)\end{array}$ & $\begin{array}{c}0,02 \\
(0,21)\end{array}$ & $\begin{array}{c}-0,09^{\star \star \star} \\
(0,00)\end{array}$ & $\begin{array}{c}-0,11^{\star * \star} \\
(0,00)\end{array}$ & $\begin{array}{c}-0,03^{* *} \\
(0,02)\end{array}$ \\
\hline & $\mathrm{Bac}+2$ ou sup. & $\begin{array}{c}-0,01 \\
(0,70)\end{array}$ & $\begin{array}{l}0,04^{\star \star} \\
(0,02)\end{array}$ & $\begin{array}{c}-0,03 \\
(0,11)\end{array}$ & $\begin{array}{c}-0,08^{\star \star *} \\
(0,00)\end{array}$ & $\begin{array}{c}-0,03^{* *} \\
(0,04)\end{array}$ \\
\hline & Non renseigné & $\begin{array}{c}-0,02 \\
(0,21)\end{array}$ & $\begin{array}{c}0,02 \\
(0,12)\end{array}$ & $\begin{array}{c}-0,01 \\
(0,34)\end{array}$ & $\begin{array}{c}-0,08^{\star \star *} \\
(0,00)\end{array}$ & $\begin{array}{c}0,00 \\
(0,86)\end{array}$ \\
\hline \multirow[t]{6}{*}{$\begin{array}{l}\text { PCS } \\
\text { Réf : Employé }\end{array}$} & Agriculteur & $\begin{array}{c}-0,22^{\star \star \star} \\
(0,00)\end{array}$ & $\begin{array}{c}-0,01 \\
(0,85)\end{array}$ & $\begin{array}{c}-0,17^{* * *} \\
(0,00)\end{array}$ & $\begin{array}{c}0,08^{*} \\
(0,08)\end{array}$ & $\begin{array}{c}0,02 \\
(0,68)\end{array}$ \\
\hline & $\begin{array}{l}\text { Artisan, commerçant, chef } \\
\text { d'entreprise }\end{array}$ & $\begin{array}{l}0,07^{\star \star \star} \\
(0,01)\end{array}$ & $\begin{array}{c}-0,02 \\
(0,51)\end{array}$ & $\begin{array}{l}0,01 \\
(0,73)\end{array}$ & $\begin{array}{c}0,00 \\
(0,98)\end{array}$ & $\begin{array}{c}-0,07^{* * *} \\
(0,01)\end{array}$ \\
\hline & Cadre et profession intellectuelle & $\begin{array}{l}0,06^{\star * *} \\
(0,00)\end{array}$ & $\begin{array}{c}-0,05^{* * *} \\
(0,01)\end{array}$ & $\begin{array}{l}0,06^{\star \star *} \\
(0,00)\end{array}$ & $\begin{array}{c}-0,07^{\star \star *} \\
(0,00)\end{array}$ & $\begin{array}{c}-0,05^{\star \star \star} \\
(0,00)\end{array}$ \\
\hline & Profession intermédiaires & $\begin{array}{l}0,01 \\
(0,70)\end{array}$ & $\begin{array}{c}0,02^{*} \\
(0,10)\end{array}$ & $\begin{array}{l}0,04^{\star \star \star} \\
(0,01)\end{array}$ & $\begin{array}{c}-0,03^{\star \star} \\
(0,03)\end{array}$ & $\begin{array}{c}-0,01 \\
(0,34)\end{array}$ \\
\hline & Ouvrier & $\begin{array}{c}-0,18^{\star \star *} \\
(0,00)\end{array}$ & $\begin{array}{l}0,07^{\star \star *} \\
(0,00)\end{array}$ & $\begin{array}{c}-0,18^{\star \star *} \\
(0,00)\end{array}$ & $\begin{array}{c}-0,07^{\star * *} \\
(0,00)\end{array}$ & $\begin{array}{c}-0,01 \\
(0,73)\end{array}$ \\
\hline & Non renseigné & $\begin{array}{c}-0,23^{\star \star \star} \\
(0,00)\end{array}$ & $\begin{array}{c}-0,01 \\
(0,65)\end{array}$ & $\begin{array}{l}-0,26^{* * *} \\
(0,00)\end{array}$ & $\begin{array}{c}0,00 \\
(0,94)\end{array}$ & $\begin{array}{l}0,03 \\
(0,11)\end{array}$ \\
\hline \multirow[t]{3}{*}{$\begin{array}{l}\text { Contrat de travail } \\
\text { Réf : Sans limitation de durée }\end{array}$} & $\begin{array}{l}\text { Durée limitée (Contrat à durée } \\
\text { déterminée ou intérim) }\end{array}$ & $\begin{array}{l}0,00 \\
(0,91)\end{array}$ & $\begin{array}{l}0,00 \\
(0,91)\end{array}$ & $\begin{array}{c}-0,04^{* *} \\
(0,02)\end{array}$ & $\begin{array}{c}0,02 \\
(0,19)\end{array}$ & $\begin{array}{l}0,04^{* *} \\
(0,03)\end{array}$ \\
\hline & Autres & $\begin{array}{c}-0,01 \\
(0,67)\end{array}$ & $\begin{array}{c}-0,02 \\
(0,26)\end{array}$ & $\begin{array}{c}-0,04 \\
(0,15)\end{array}$ & $\begin{array}{r}0,05^{*} \\
(0,07)\end{array}$ & $\begin{array}{l}0,06^{\star \star \star} \\
(0,01)\end{array}$ \\
\hline & Indépendant & $\begin{array}{l}0,01 \\
(0,71)\end{array}$ & $\begin{array}{l}0,01 \\
(0,60)\end{array}$ & $\begin{array}{l}0,01 \\
(0,68)\end{array}$ & $\begin{array}{c}-0,15^{\star \star \star} \\
(0,00)\end{array}$ & $\begin{array}{c}-0,15^{\star \star \star} \\
(0,00)\end{array}$ \\
\hline $\begin{array}{l}\text { Temps partiel } \\
\text { Réf : Non }\end{array}$ & Oui & $\begin{array}{c}-0,01 \\
(0,39)\end{array}$ & $\begin{array}{c}-0,01 \\
(0,68)\end{array}$ & $\begin{array}{c}-0,02 \\
(0,12)\end{array}$ & $\begin{array}{l}0,04^{\star \star \star} \\
(0,00)\end{array}$ & $\begin{array}{c}-0,02 \\
(0,24)\end{array}$ \\
\hline $\begin{array}{l}\text { Temps de travail souvent } \\
\text { supérieur à } 48 \mathrm{~h} \text { par semaine } \\
\text { Réf : Non }\end{array}$ & Oui & $\begin{array}{l}0,12^{\star \star \star} \\
(0,00)\end{array}$ & $\begin{array}{l}0,10^{\star \star \star} \\
(0,00)\end{array}$ & $\begin{array}{l}0,09^{\star \star \star} \\
(0,00)\end{array}$ & $\begin{array}{c}-0,04^{\star \star \star} \\
(0,00)\end{array}$ & $\begin{array}{c}-0,04^{\star \star *} \\
(0,00)\end{array}$ \\
\hline $\begin{array}{l}\text { Travail posté } \\
\text { Réf : Non }\end{array}$ & Oui & $\begin{array}{c}-0,02 \\
(0,19)\end{array}$ & $\begin{array}{l}0,07^{\star \star *} \\
(0,00)\end{array}$ & $\begin{array}{c}0,02 \\
(0,17)\end{array}$ & $\begin{array}{l}0,03^{\star * *} \\
(0,01)\end{array}$ & $\begin{array}{r}0,02^{*} \\
(0,06)\end{array}$ \\
\hline $\begin{array}{l}\text { Travail de nuit } \\
\text { Réf : Non }\end{array}$ & Oui & $\begin{array}{l}0,05^{\star \star *} \\
(0,00)\end{array}$ & $\begin{array}{l}0,06^{\star \star *} \\
(0,00)\end{array}$ & $\begin{array}{c}0,01 \\
(0,37)\end{array}$ & $\begin{array}{l}0,03^{* *} \\
(0,02)\end{array}$ & $\begin{array}{l}0,07^{\star * *} \\
(0,00)\end{array}$ \\
\hline $\begin{array}{l}\text { Travail répétitif ou travail à la } \\
\text { chaine } \\
\text { Réf : Non }\end{array}$ & Oui & $\begin{array}{l}0,07^{\star \star *} \\
(0,00)\end{array}$ & $\begin{array}{l}0,03^{\star \star *} \\
(0,00)\end{array}$ & $\begin{array}{l}0,04^{\star \star \star} \\
(0,00)\end{array}$ & $\begin{array}{l}0,09^{\star \star \star} \\
(0,00)\end{array}$ & $\begin{array}{l}0,11^{\star \star *} \\
(0,00)\end{array}$ \\
\hline $\begin{array}{l}\text { Travail physiquement exigeant } \\
\text { Réf: Non }\end{array}$ & Oui & $\begin{array}{l}0,09^{* * *} \\
(0,00)\end{array}$ & $\begin{array}{l}0,17^{\star \star *} \\
(0,00)\end{array}$ & $\begin{array}{l}0,11^{\star \star \star} \\
(0,00)\end{array}$ & $\begin{array}{l}0,03^{\star \star \star} \\
(0,01)\end{array}$ & $\begin{array}{c}0,06 \\
(0,00)\end{array}$ \\
\hline
\end{tabular}


Tableau A (suite)

\begin{tabular}{|c|c|c|c|c|c|c|}
\hline & & \multicolumn{2}{|c|}{$\begin{array}{c}\text { DIM } 4 \\
\text { Rapports sociaux, } \\
\text { relations de travail }\end{array}$} & \multicolumn{2}{|c|}{$\begin{array}{c}\text { DIM } 5 \\
\text { Conflits de valeurs }\end{array}$} & \multirow{2}{*}{$\begin{array}{c}\text { DIM } 6 \\
\text { Insécurité } \\
\text { socio-éco } \\
\text { RPS } 15\end{array}$} \\
\hline & & RPS 11 & RPS 12 & RPS 13 & RPS 14 & \\
\hline \multicolumn{2}{|l|}{ Indicatrices du secteur d'activité } & Oui & Oui & Oui & Oui & Oui \\
\hline $\begin{array}{l}\text { Sexe } \\
\text { Réf : Homme }\end{array}$ & Femme & $\begin{array}{l}0,01 \\
(0,60)\end{array}$ & $\begin{array}{r}-0,01 \\
(0,21)\end{array}$ & $\begin{array}{l}-0,03^{\star \star \star} \\
(0,01)\end{array}$ & $\begin{array}{c}-0,01 \\
(0,40)\end{array}$ & $\begin{array}{l}0,04^{\star \star \star} \\
(0,00)\end{array}$ \\
\hline \multirow[t]{3}{*}{$\begin{array}{l}\text { Age } \\
\text { Réf : }[35 ; 44]\end{array}$} & {$[-; 34]$} & $\begin{array}{c}0,01 \\
(0,35)\end{array}$ & $\begin{array}{c}-0,02 \\
(0,11)\end{array}$ & $\begin{array}{c}-0,01 \\
(0,24)\end{array}$ & $\begin{array}{r}-0,01 \\
(0,58)\end{array}$ & $\begin{array}{l}-0,03^{\star \star \star} \\
(0,00)\end{array}$ \\
\hline & {$[45 ; 54]$} & $\begin{array}{c}-0,03^{\star \star} \\
(0,03)\end{array}$ & $\begin{array}{c}-0,01 \\
(0,16)\end{array}$ & $\begin{array}{l}-0,03^{* \star *} \\
(0,01)\end{array}$ & $\begin{array}{l}-0,01 \\
(0,21)\end{array}$ & $\begin{array}{c}0,00 \\
(0,74)\end{array}$ \\
\hline & {$[55 ;+]$} & $\begin{array}{c}-0,07^{\star \star \star} \\
(0,00)\end{array}$ & $\begin{array}{l}-0,04^{\star \star *} \\
(0,00)\end{array}$ & $\begin{array}{l}-0,06^{\star \star *} \\
(0,00)\end{array}$ & $\begin{array}{l}-0,06^{\star * *} \\
(0,00)\end{array}$ & $\begin{array}{l}-0,10^{\star \star \star} \\
(0,00)\end{array}$ \\
\hline \multirow[t]{4}{*}{$\begin{array}{l}\text { Education } \\
\text { Ref : Baccalauréat }\end{array}$} & Aucun dipl., CEP, brevet, BEPC & $\begin{array}{c}-0,09^{\star \star \star} \\
(0,00)\end{array}$ & $\begin{array}{l}-0,08^{\star * *} \\
(0,00)\end{array}$ & $\begin{array}{l}-0,09^{\star \star *} \\
(0,00)\end{array}$ & $\begin{array}{l}-0,14^{\star * *} \\
(0,00)\end{array}$ & $\begin{array}{l}0,01 \\
(0,70)\end{array}$ \\
\hline & CAP, BEP & $\begin{array}{c}-0,07^{\star \star \star} \\
(0,00)\end{array}$ & $\begin{array}{l}-0,07^{\star \star \star} \\
(0,00)\end{array}$ & $\begin{array}{r}-0,03^{\star} \\
(0,06)\end{array}$ & $\begin{array}{l}-0,11^{\star \star \star} \\
(0,00)\end{array}$ & $\begin{array}{c}-0,02 \\
(0,24)\end{array}$ \\
\hline & Bac+2 ou sup. & $\begin{array}{r}-0,03^{\star} \\
(0,09)\end{array}$ & $\begin{array}{l}-0,04^{\star \star \star} \\
(0,00)\end{array}$ & $\begin{array}{c}0,00 \\
(0,89)\end{array}$ & $\begin{array}{l}-0,08^{\star \star \star} \\
(0,00)\end{array}$ & $\begin{array}{c}-0,01 \\
(0,39)\end{array}$ \\
\hline & Non renseigné & $\begin{array}{c}-0,06^{\star * \star} \\
(0,00)\end{array}$ & $\begin{array}{l}-0,05^{\star \star *} \\
(0,00)\end{array}$ & $\begin{array}{c}-0,03^{\star *} \\
(0,02)\end{array}$ & $\begin{array}{l}-0,09^{\star \star *} \\
(0,00)\end{array}$ & $\begin{array}{c}0,01 \\
(0,61)\end{array}$ \\
\hline \multirow[t]{6}{*}{$\begin{array}{l}\text { PCS } \\
\text { Réf : Employé }\end{array}$} & Agriculteur & $\begin{array}{l}0,19^{\star \star \star} \\
(0,00)\end{array}$ & $\begin{array}{l}0,08^{*} \\
(0,08)\end{array}$ & $\begin{array}{c}0,06 \\
(0,19)\end{array}$ & $\begin{array}{l}0,17^{\star \star \star} \\
(0,00)\end{array}$ & $\begin{array}{c}-0,01 \\
(0,77)\end{array}$ \\
\hline & $\begin{array}{l}\text { Artisan, commerçant, chef } \\
\text { d'entreprise }\end{array}$ & $\begin{array}{r}-0,02 \\
(0,56)\end{array}$ & $\begin{array}{c}0,03 \\
(0,32)\end{array}$ & $\begin{array}{l}0,05 \\
(0,12)\end{array}$ & $\begin{array}{l}0,02 \\
(0,48)\end{array}$ & $\begin{array}{l}0,05^{*} \\
(0,06)\end{array}$ \\
\hline & Cadre et profession intellectuelle & $\begin{array}{c}-0,02 \\
(0,37)\end{array}$ & $\begin{array}{l}0,04^{* \star *} \\
(0,01)\end{array}$ & $\begin{array}{l}0,04^{\star *} \\
(0,04)\end{array}$ & $\begin{array}{l}0,04^{\star *} \\
(0,03)\end{array}$ & $\begin{array}{c}0,02 \\
(0,35)\end{array}$ \\
\hline & Profession intermédiaires & $\begin{array}{r}-0,01 \\
(0,62)\end{array}$ & $\begin{array}{c}0,00 \\
(0,92)\end{array}$ & $\begin{array}{l}0,04^{\star \star *} \\
(0,01)\end{array}$ & $\begin{array}{l}0,03^{\star \star} \\
(0,04)\end{array}$ & $\begin{array}{l}0,01 \\
(0,29)\end{array}$ \\
\hline & Ouvrier & $\begin{array}{l}-0,07^{\star \star \star} \\
(0,00)\end{array}$ & $\begin{array}{l}-0,03^{\star * *} \\
(0,01)\end{array}$ & $\begin{array}{l}-0,07^{\star * \star} \\
(0,00)\end{array}$ & $\begin{array}{l}-0,07^{\star * \star} \\
(0,00)\end{array}$ & $\begin{array}{c}0,00 \\
(0,87)\end{array}$ \\
\hline & Non renseigné & $\begin{array}{l}-0,08^{\star \star \star} \\
(0,00)\end{array}$ & $\begin{array}{r}-0,03 \\
(0,11)\end{array}$ & $\begin{array}{l}-0,10^{\star \star \star} \\
(0,00)\end{array}$ & $\begin{array}{l}-0,05^{\star \star \star} \\
(0,01)\end{array}$ & $\begin{array}{l}0,00 \\
(0,81)\end{array}$ \\
\hline \multirow[t]{3}{*}{$\begin{array}{l}\text { Contrat de travail } \\
\text { Réf: Sans limitation de durée }\end{array}$} & $\begin{array}{l}\text { Durée limitée (Contrat à durée } \\
\text { déterminée ou intérim) }\end{array}$ & $\begin{array}{c}-0,04^{\star *} \\
(0,02)\end{array}$ & $\begin{array}{r}-0,02 \\
(0,29)\end{array}$ & $\begin{array}{l}-0,05^{\star \star \star} \\
(0,00)\end{array}$ & $\begin{array}{r}-0,03^{*} \\
(0,09)\end{array}$ & $\begin{array}{l}0,10^{\star \star \star} \\
(0,00)\end{array}$ \\
\hline & Autres (saisonniers...) & $\begin{array}{r}-0,02 \\
(0,51)\end{array}$ & $\begin{array}{r}-0,01 \\
(0,60)\end{array}$ & $\begin{array}{r}-0,04^{*} \\
(0,06)\end{array}$ & $\begin{array}{c}0,02 \\
(0,37)\end{array}$ & $\begin{array}{l}0,11^{\star \star \star} \\
(0,00)\end{array}$ \\
\hline & Indépendant & $\begin{array}{l}-0,12^{\star \star \star} \\
(0,00)\end{array}$ & $\begin{array}{l}-0,15^{\star \star \star} \\
(0,00)\end{array}$ & $\begin{array}{l}-0,14^{\star * \star} \\
(0,00)\end{array}$ & $\begin{array}{l}-0,21^{\star * \star} \\
(0,00)\end{array}$ & $\begin{array}{l}0,00 \\
(0,84)\end{array}$ \\
\hline $\begin{array}{l}\text { Temps partiel } \\
\text { Réf : Non }\end{array}$ & Oui & $\begin{array}{l}0,00 \\
(0,91)\end{array}$ & $\begin{array}{c}-0,02^{\star \star} \\
(0,04)\end{array}$ & $\begin{array}{l}0,00 \\
(0,91)\end{array}$ & $\begin{array}{l}0,01 \\
(0,30)\end{array}$ & $\begin{array}{c}0,01 \\
(0,32)\end{array}$ \\
\hline $\begin{array}{l}\text { Temps de travail souvent } \\
\text { supérieur à } 48 \mathrm{~h} \\
\text { Réf : Non }\end{array}$ & Oui & $\begin{array}{l}0,01 \\
(0,57)\end{array}$ & $\begin{array}{l}0,03^{\star \star *} \\
(0,01)\end{array}$ & $\begin{array}{l}0,06^{\star \star *} \\
(0,00)\end{array}$ & $\begin{array}{r}-0,01 \\
(0,55)\end{array}$ & $\begin{array}{l}0,03^{\star \star *} \\
(0,01)\end{array}$ \\
\hline $\begin{array}{l}\text { Travail posté } \\
\text { Réf : Non }\end{array}$ & Oui & $\begin{array}{l}0,01 \\
(0,66)\end{array}$ & $\begin{array}{l}0,01 \\
(0,59)\end{array}$ & $\begin{array}{l}0,04^{\star \star *} \\
(0,00)\end{array}$ & $\begin{array}{c}0,02 \\
(0,22)\end{array}$ & $\begin{array}{l}0,04^{\star \star *} \\
(0,00)\end{array}$ \\
\hline $\begin{array}{l}\text { Travail de nuit } \\
\text { Réf : Non }\end{array}$ & Oui & $\begin{array}{c}0,02 \\
(0,10)\end{array}$ & $\begin{array}{c}0,01 \\
(0,59)\end{array}$ & $\begin{array}{c}0,01 \\
(0,44)\end{array}$ & $\begin{array}{c}0,02 \\
(0,18)\end{array}$ & $\begin{array}{c}0,02 \\
(0,18)\end{array}$ \\
\hline $\begin{array}{l}\text { Travail répétitif ou à la chaine } \\
\text { Réf : Non }\end{array}$ & Oui & $\begin{array}{l}0,11^{\star \star \star} \\
(0,00)\end{array}$ & $\begin{array}{l}0,06^{\star \star \star} \\
(0,00)\end{array}$ & $\begin{array}{l}0,08^{\star \star \star} \\
(0,00)\end{array}$ & $\begin{array}{l}0,08^{\star \star \star} \\
(0,00)\end{array}$ & $\begin{array}{l}0,08^{\star \star \star} \\
(0,00)\end{array}$ \\
\hline $\begin{array}{l}\text { Travail physiquement exigeant } \\
\text { Réf : Non }\end{array}$ & Oui & $\begin{array}{l}0,08^{\star \star *} \\
(0,00)\end{array}$ & $\begin{array}{l}0,03^{\star \star *} \\
(0,00)\end{array}$ & $\begin{array}{l}0,07^{\star \star \star} \\
(0,00)\end{array}$ & $\begin{array}{l}0,06^{\star \star *} \\
(0,00)\end{array}$ & $\begin{array}{l}0,03^{\star \star *} \\
(0,00)\end{array}$ \\
\hline
\end{tabular}

Note : les effets marginaux ont été calculés à partir des résultats d'estimation de la spécification 1 du modèle (cf. encadré 3), estimé sur l'échantillon incluant les individus enquêtés en 2006 ou 2010. Les effets marginaux moyens présentés dans le tableau ont cependant été calculés sur les individus de 2006 uniquement. Les écart-types et probabilités critiques associées ont été calculés par la méthode du delta.

Lecture : le tableau présente les effets marginaux moyens des caractéristiques individuelles sur l'exposition aux RPS. Par hypothèse, l'effet des caractéristiques est supposé identique en 2006 et 2010. Par exemple, toutes choses égales par ailleurs, les individus en emploi âgés de 34 ans ou moins connaissent en moyenne un risque d'être exposé au RPS 1 (travailler sous pression) inférieur de 0,03 point de pourcentage au risque des individus âgés de 35 à 44 ans. Les nombres entre parenthèses correspondent aux probabilités critiques associées aux tests de nullité des effets marginaux : * significatif à $10 \%$, ** significatif à $5 \%$, *** significatif à $1 \%$.

Champ : individus âgés de 20 à 68 ans, déclarant exercer une activité professionnelle en tant que salarié d'une entreprise, d'un artisan, d'une association, d'un particulier, ou en tant que chef d'entreprise ou indépendant, enquêtés en 2006 ou 2010.

Source : SIP 2006 et 2010. 
Tableau B

Effets marginaux des caractéristiques individuelles sur l'évolution de l'exposition aux RPS entre 2006 et 2010

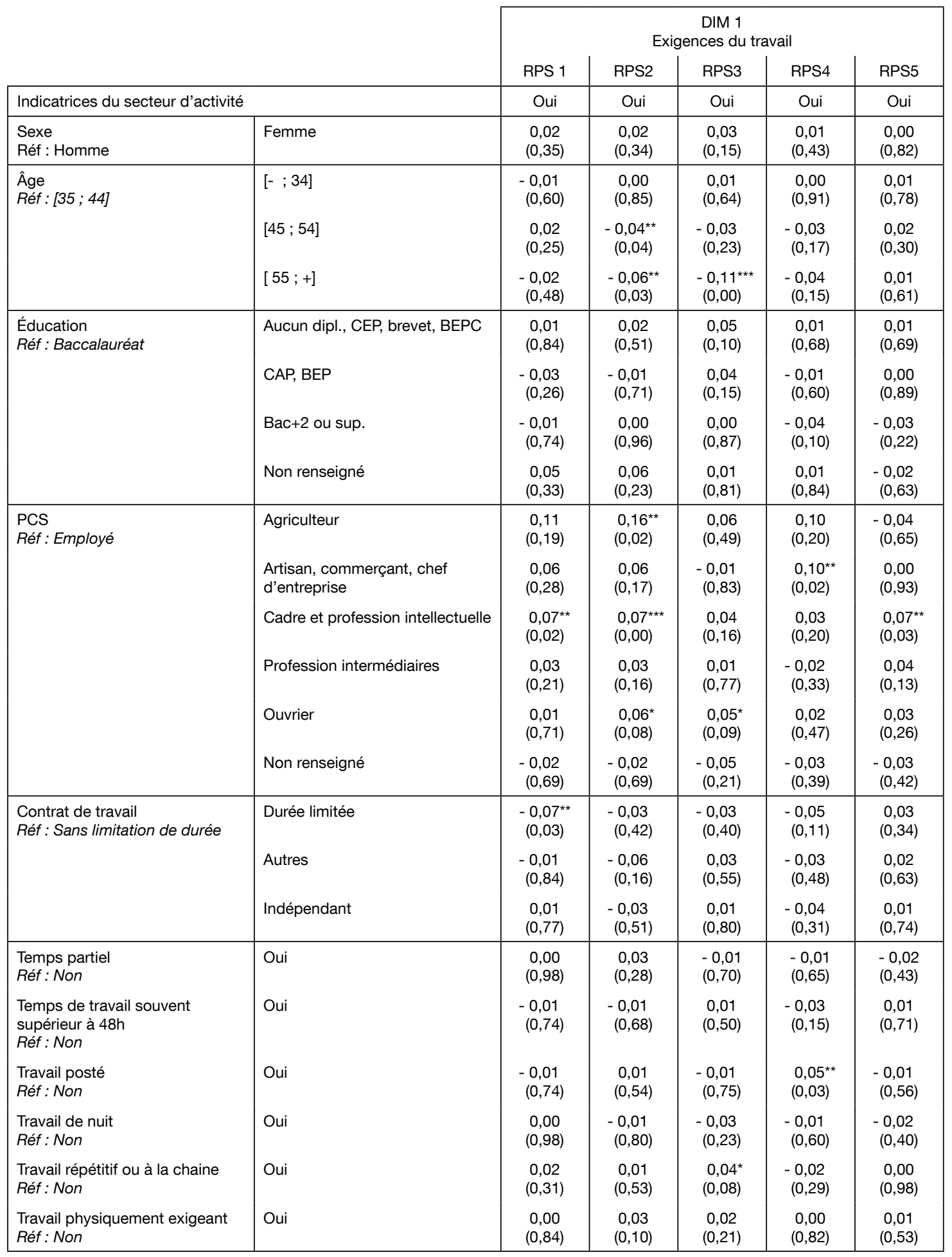


Tableau B (suite)

\begin{tabular}{|c|c|c|c|c|c|c|}
\hline & \multirow{2}{*}{\multicolumn{3}{|c|}{$\begin{array}{c}\text { DIM } 2 \\
\text { Exigences émotionnelles }\end{array}$}} & & \\
\hline & & & & & \multicolumn{2}{|c|}{$\begin{array}{c}\text { DIM } 3 \\
\text { Manque d'autonomie } \\
\text { et marges } \\
\text { de manœuvre }\end{array}$} \\
\hline & & \multirow{2}{*}{$\begin{array}{c}\text { RPS6 } \\
\text { Oui }\end{array}$} & \multirow{2}{*}{$\begin{array}{c}\text { RPS7 } \\
\text { Oui }\end{array}$} & \multirow{2}{*}{$\begin{array}{c}\text { RPS8 } \\
\text { Oui }\end{array}$} & \multirow{2}{*}{$\begin{array}{c}\text { RPS } 9 \\
\text { Oui }\end{array}$} & \multirow{2}{*}{$\begin{array}{c}\text { RPS10 } \\
\text { Oui }\end{array}$} \\
\hline Indicatrices du secteur d'activit & & & & & & \\
\hline $\begin{array}{l}\text { Sexe } \\
\text { Réf: Homme }\end{array}$ & femme & $\begin{array}{c}0,00 \\
(1,00)\end{array}$ & $\begin{array}{l}0,01 \\
(0,44)\end{array}$ & $\begin{array}{l}-0,01 \\
(0,60)\end{array}$ & $\begin{array}{c}0,00 \\
(0,82)\end{array}$ & $\begin{array}{c}0,02 \\
(0,42)\end{array}$ \\
\hline \multirow[t]{3}{*}{$\begin{array}{l}\text { Âge } \\
\text { Réf : [35; 44] }\end{array}$} & & $\begin{array}{c}-0,03 \\
(0,13)\end{array}$ & $\begin{array}{l}0,00 \\
(0,90)\end{array}$ & $\begin{array}{l}0,01 \\
(0,80)\end{array}$ & $\begin{array}{c}0,01 \\
(0,71)\end{array}$ & $\begin{array}{l}0,06^{\star *} \\
(0,02)\end{array}$ \\
\hline & {$[45 ; 54]$} & $\begin{array}{c}-0,05^{\star \star} \\
(0,02)\end{array}$ & $\begin{array}{c}-0,04^{\star *} \\
(0,05)\end{array}$ & $\begin{array}{c}-0,01 \\
(0,77)\end{array}$ & $\begin{array}{c}0,03 \\
(0,12)\end{array}$ & $\begin{array}{c}0,00 \\
(0,86)\end{array}$ \\
\hline & {$[55 ;+]$} & $\begin{array}{r}-0,05^{*} \\
(0,06)\end{array}$ & $\begin{array}{c}-0,02 \\
(0,36)\end{array}$ & $\begin{array}{c}0,00 \\
(0,99)\end{array}$ & $\begin{array}{c}0,00 \\
(0,98)\end{array}$ & $\begin{array}{c}0,03 \\
(0,27)\end{array}$ \\
\hline \multirow[t]{4}{*}{$\begin{array}{l}\text { Éducation } \\
\text { Réf : Baccalauréat }\end{array}$} & Aucun dipl., CEP, brevet, BEPC & $\begin{array}{c}0,00 \\
(0,97)\end{array}$ & $\begin{array}{c}-0,02 \\
(0,51)\end{array}$ & $\begin{array}{c}0,02 \\
(0,44)\end{array}$ & $\begin{array}{c}0,02 \\
(0,53)\end{array}$ & $\begin{array}{c}0,00 \\
(0,96)\end{array}$ \\
\hline & CAP, BEP & $\begin{array}{r}-0,01 \\
(0,55)\end{array}$ & $\begin{array}{c}-0,02 \\
(0,39)\end{array}$ & $\begin{array}{c}-0,02 \\
(0,52)\end{array}$ & $\begin{array}{l}0,06^{* * *} \\
(0,01)\end{array}$ & $\begin{array}{l}-0,01 \\
(0,81)\end{array}$ \\
\hline & $\mathrm{Bac}+2$ ou sup. & $\begin{array}{r}-0,02 \\
(0,39)\end{array}$ & $\begin{array}{c}-0,02 \\
(0,50)\end{array}$ & $\begin{array}{r}-0,02 \\
(0,42)\end{array}$ & $\begin{array}{l}0,07^{\star \star \star} \\
(0,01)\end{array}$ & $\begin{array}{c}0,00 \\
(0,90)\end{array}$ \\
\hline & Non renseigné & $\begin{array}{c}-0,02 \\
(0,61)\end{array}$ & $\begin{array}{c}0,00 \\
(1,00)\end{array}$ & $\begin{array}{c}0,01 \\
(0,77)\end{array}$ & $\begin{array}{c}0,02 \\
(0,72)\end{array}$ & $\begin{array}{c}-0,07 \\
(0,17)\end{array}$ \\
\hline \multirow[t]{6}{*}{$\begin{array}{l}\text { PCS } \\
\text { Réf : Employé }\end{array}$} & Agriculteur & $\begin{array}{c}0,00 \\
(0,98)\end{array}$ & $\begin{array}{c}-0,02 \\
(0,78)\end{array}$ & $\begin{array}{c}-0,01 \\
(0,86)\end{array}$ & $\begin{array}{c}0,09 \\
(0,34)\end{array}$ & $\begin{array}{c}-0,09 \\
(0,33)\end{array}$ \\
\hline & $\begin{array}{l}\text { Artisan, commerçant, chef } \\
\text { d'entreprise }\end{array}$ & $\begin{array}{c}0,03 \\
(0,58)\end{array}$ & $\begin{array}{c}0,01 \\
(0,86)\end{array}$ & $\begin{array}{r}-0,02 \\
(0,78)\end{array}$ & $\begin{array}{c}0,09 \\
(0,11)\end{array}$ & $\begin{array}{r}-0,01 \\
(0,92)\end{array}$ \\
\hline & Cadre et profession intellectuelle & $\begin{array}{r}-0,02 \\
(0,53)\end{array}$ & $\begin{array}{c}0,00 \\
(0,93)\end{array}$ & $\begin{array}{r}-0,01 \\
(0,78)\end{array}$ & $\begin{array}{c}0,01 \\
(0,85)\end{array}$ & $\begin{array}{c}0,03 \\
(0,32)\end{array}$ \\
\hline & Profession intermédiaires & $\begin{array}{c}-0,06^{\star *} \\
(0,03)\end{array}$ & $\begin{array}{r}-0,03 \\
(0,16)\end{array}$ & $\begin{array}{c}-0,04 \\
(0,16)\end{array}$ & $\begin{array}{l}0,06^{\star \star} \\
(0,02)\end{array}$ & $\begin{array}{l}0,07^{\star \star \star} \\
(0,01)\end{array}$ \\
\hline & Ouvrier & $\begin{array}{c}0,00 \\
(0,97)\end{array}$ & $\begin{array}{c}-0,03 \\
(0,29)\end{array}$ & $\begin{array}{c}-0,03 \\
(0,30)\end{array}$ & $\begin{array}{c}0,01 \\
(0,79)\end{array}$ & $\begin{array}{l}0,02 \\
(0,49)\end{array}$ \\
\hline & Non renseigné & $\begin{array}{r}-0,03 \\
(0,42)\end{array}$ & $\begin{array}{c}-0,02 \\
(0,65)\end{array}$ & $\begin{array}{r}-0,02 \\
(0,53)\end{array}$ & $\begin{array}{c}0,01 \\
(0,82)\end{array}$ & $\begin{array}{c}-0,02 \\
(0,57)\end{array}$ \\
\hline \multirow[t]{3}{*}{$\begin{array}{l}\text { Contrat de travail } \\
\text { Réf: Sans limitation de durée }\end{array}$} & Durée limitée & $\begin{array}{c}0,01 \\
(0,66)\end{array}$ & $\begin{array}{c}-0,05 \\
(0,15)\end{array}$ & $\begin{array}{c}-0,07^{\star *} \\
(0,04)\end{array}$ & $\begin{array}{c}-0,04 \\
(0,23)\end{array}$ & $\begin{array}{c}-0,05 \\
(0,20)\end{array}$ \\
\hline & Autres & $\begin{array}{r}-0,05 \\
(0,26)\end{array}$ & $\begin{array}{r}-0,03 \\
(0,55)\end{array}$ & $\begin{array}{r}-0,02 \\
(0,72)\end{array}$ & $\begin{array}{l}0,09^{\star *} \\
(0,07)\end{array}$ & $\begin{array}{l}-0,01 \\
(0,85)\end{array}$ \\
\hline & Indépendant & $\begin{array}{c}0,06 \\
(0,19)\end{array}$ & $\begin{array}{r}-0,03 \\
(0,39)\end{array}$ & $\begin{array}{c}0,03 \\
(0,52)\end{array}$ & $\begin{array}{c}-0,01 \\
(0,87)\end{array}$ & $\begin{array}{c}0,04 \\
(0,33)\end{array}$ \\
\hline $\begin{array}{l}\text { Temps partiel } \\
\text { Réf : Non }\end{array}$ & Oui & $\begin{array}{c}-0,01 \\
(0,73)\end{array}$ & $\begin{array}{l}0,00 \\
(0,91)\end{array}$ & $\begin{array}{c}0,00 \\
(0,94)\end{array}$ & $\begin{array}{c}0,04 \\
(0,12)\end{array}$ & $\begin{array}{l}0,01 \\
(0,70)\end{array}$ \\
\hline $\begin{array}{l}\text { Temps de travail souvent } \\
\text { supérieur à } 48 \mathrm{~h} \\
\text { Réf : Non }\end{array}$ & Oui & $\begin{array}{r}-0,04^{*} \\
(0,07)\end{array}$ & $\begin{array}{c}-0,01 \\
(0,63)\end{array}$ & $\begin{array}{c}0,00 \\
(0,97)\end{array}$ & $\begin{array}{c}0,03 \\
(0,15)\end{array}$ & $\begin{array}{l}0,03 \\
(0,17)\end{array}$ \\
\hline $\begin{array}{l}\text { Travail posté } \\
\text { Réf : Non }\end{array}$ & Oui & $\begin{array}{r}-0,03 \\
(0,25)\end{array}$ & $\begin{array}{c}-0,02 \\
(0,47)\end{array}$ & $\begin{array}{r}-0,03 \\
(0,21)\end{array}$ & $\begin{array}{c}0,02 \\
(0,33)\end{array}$ & $\begin{array}{c}-0,02 \\
(0,44)\end{array}$ \\
\hline $\begin{array}{l}\text { Travail de nuit } \\
\text { Réf : Non }\end{array}$ & Oui & $\begin{array}{r}0,04^{*} \\
(0,09)\end{array}$ & $\begin{array}{c}0,02 \\
(0,43)\end{array}$ & $\begin{array}{c}0,02 \\
(0,49)\end{array}$ & $\begin{array}{r}-0,02 \\
(0,36)\end{array}$ & $\begin{array}{c}-0,02 \\
(0,42)\end{array}$ \\
\hline $\begin{array}{l}\text { Travail répétitif ou à la chaine } \\
\text { Réf : Non }\end{array}$ & Oui & $\begin{array}{c}0,02 \\
(0,26)\end{array}$ & $\begin{array}{c}0,00 \\
(0,48)\end{array}$ & $\begin{array}{l}0,01 \\
(0,47)\end{array}$ & $\begin{array}{c}-0,01 \\
(0,48)\end{array}$ & $\begin{array}{l}0,06^{\star \star \star} \\
(0,01)\end{array}$ \\
\hline $\begin{array}{l}\text { Travail physiquement exigeant } \\
\text { Réf : Non }\end{array}$ & Oui & $\begin{array}{c}-0,02 \\
(0,22)\end{array}$ & $\begin{array}{c}0,01 \\
(0,50)\end{array}$ & $\begin{array}{c}0,03 \\
(0,10)\end{array}$ & $\begin{array}{r}-0,03 \\
(0,12)\end{array}$ & $\begin{array}{c}-0,02 \\
(0,36)\end{array}$ \\
\hline
\end{tabular}


Tableau B (suite)

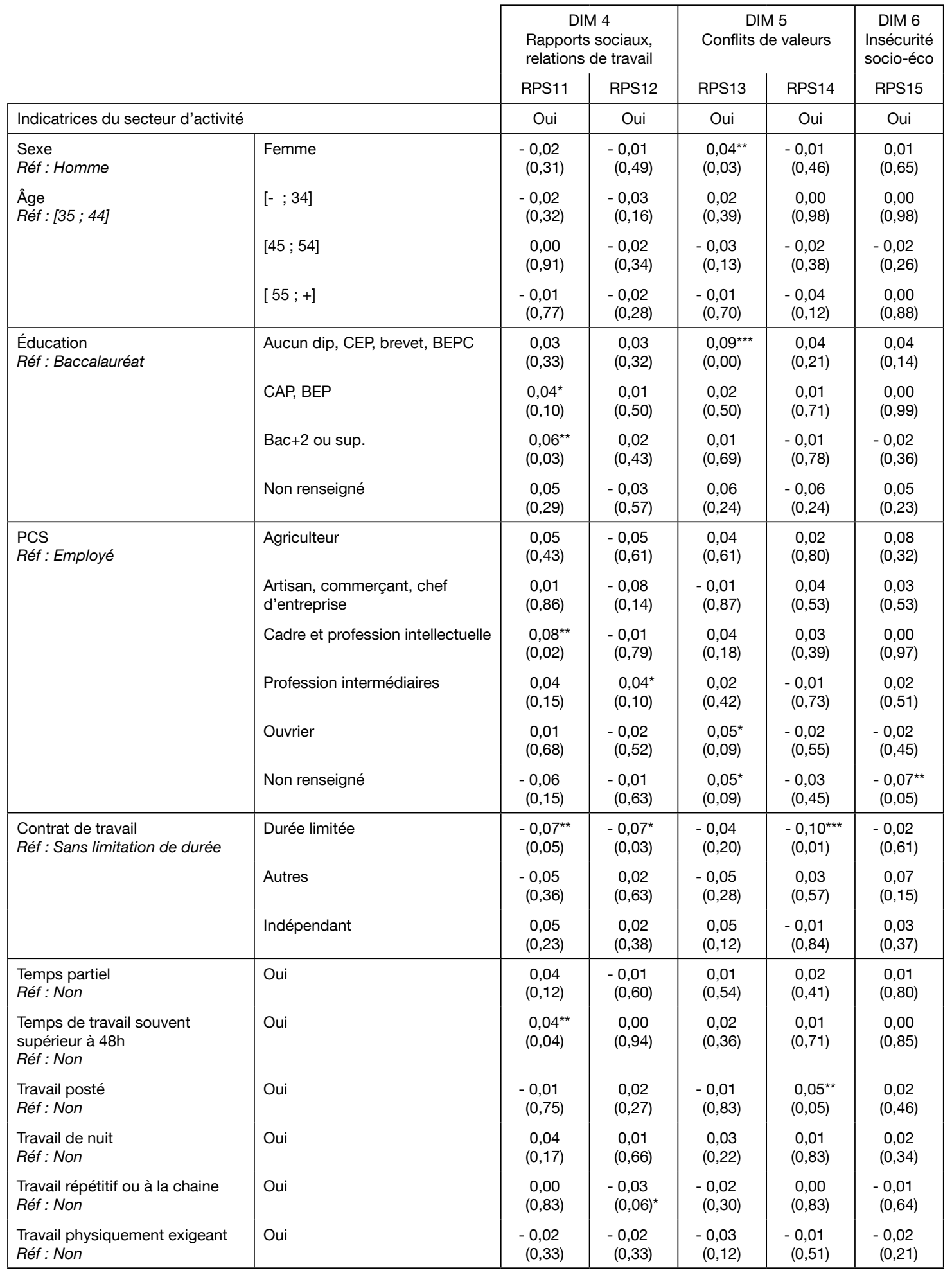

Note : les effets marginaux sont tirés des résultats d'estimation de la spécification 2 du modèle, estimé sur l'échantillon incluant les individus enquêtés en 2006 ou 2010 (cf encadré 3). Les effets marginaux moyens présentés dans le tableau ont cependant été calculés sur les individus de 2006 uniquement. Les écart-types et probabilités critiques associées ont été calculés par la méthode du delta.

Lecture : le tableau présente les effets marginaux moyens des caractéristiques individuelles sur la variation de l'exposition aux différents RPS entre 2006 et 2010. Par exemple, toutes choses égales par ailleurs, les femmes connaissent en moyenne une augmentation du risque d'être exposé au RPS 1 (travailler sous pression) supérieur de 0,02 à l'augmentation du risque que connaissent les hommes. La différence n'est cependant pas significative, ce qui indique que le sexe n'a aucune influence, toutes choses égales par ailleurs, sur l'évolution de l'exposition au RPS 1 (travailler sous pression). Les nombres entre parenthèses correspondent aux probabilités critiques associées aux tests de nullité des effets marginaux : * significatif à $10 \%$, ** significatif à $5 \%$, ,** significatif à $1 \%$.

Champ : individus âgés de 20 à 68 ans, déclarant exercer une activité professionnelle en tant que salarié d'une entreprise, d'un artisan, d'une association, d'un particulier, ou en tant que chef d'entreprise ou indépendant, enquêtés en 2006 ou 2010. Source : SIP 2006 et 2010. 